\title{
The host phenotype and microbiome varies with infection status, parasite origin and parasite microbiome composition
}

\section{Megan Hahn}

Stony Brook University https://orcid.org/0000-0001-9266-8232

Agnes Piecyk

Max Planck Institute for Evolutionary Biology: Max-Planck-Institut fur Evolutionsbiologie

Fatima Jorge

University of Otago

Robert Cerrato

Stony Brook University

\section{Martin Kalbe}

Max Planck Institute for Evolutionary Biology: Max-Planck-Institut fur Evolutionsbiologie

Nolwenn M. Dheilly ( $\square$ nolwenn.dheilly@stonybrook.edu )

Stony Brook University https://orcid.org/0000-0002-3675-5013

\section{Research}

Keywords: helminths, tripartite interactions, parasite, microbes, hosts

Posted Date: April 15th, 2021

DOI: https://doi.org/10.21203/rs.3.rs-323107/v1

License: (9) (i) This work is licensed under a Creative Commons Attribution 4.0 International License. Read Full License 
1 The host phenotype and microbiome varies with infection status, parasite origin and parasite

2 microbiome composition

4 Running title: Host-helminth interaction and the microbiome

5 Megan A Hahn ${ }^{\mathrm{a}, 1}$, Agnes Piecyk ${ }^{\mathrm{b}, \mathrm{c}, 2}$, Fátima Jorge ${ }^{\mathrm{d}, 3}$, Robert Cerrato $^{\mathrm{a}, 4}$, Martin Kalbe $^{\mathrm{b}, \mathrm{c}}$,

$6 \quad$ Nolwenn M Dheilly ${ }^{\mathrm{a}, \mathrm{e}, 5^{*}}$

$7 \quad{ }^{a}$ School of Marine and Atmospheric Sciences, Stony Brook University, Stony Brook NY, USA

$8 \quad{ }^{b}$ Evolutionary Ecology, Max Planck Institute for Evolutionary Biology, Plön, Germany

$9{ }^{c}$ Evolutionary Ecology of Marine Fishes, GEOMAR Helmholtz, Centre for Ocean Research Kiel,

10 Germany

$11{ }^{d}$ Department of Zoology, University of Otago, P.O. Box 56, Dunedin 9054, New Zealand

12 e ANSES, Agence Nationale de Sécurité Sanitaire de l'Alimentation, de l'Environnement et du

13 Travail - Laboratoire de Ploufragan-Plouzané, Unité Génétique Virale de Biosécurité,

14 Ploufragan, France

15 ํㅡㄹahn@health.nyc.gov, ${ }^{2}$ apiecyk@evolbio.mpg.de, ${ }^{3}$ fatima.esperanca@gmail.com,

16 4 ${ }_{\text {rcerrato@ } \text { stonybrook.edu, }}^{5}$ nolwenn.dheilly@anses.fr

17 Corresponding author* 
Background: A growing literature demonstrates the impact of helminths on their host gut

21 microbiome. However, there is now a need to investigate helminth associated microbes and the

22 complex tripartite interactions between parasite, microbes, and hosts.

23 Methods: We investigated whether the stickleback host microbiome depends on eco-evolutionary

24 variables by testing the impact of exposure to the parasite Schistocephalus solidus, infection

25 success, host genotype, parasite genotype, and parasite microbiome composition.

26 Results: We observed constitutive differences in the microbiome of stickleback of different origin

27 that increased when sticklebacks exposed to the parasite resisted infection. In contrast, the 28 microbiome of successfully infected sticklebacks varies with parasite genotype. More specifically,

29 we reveal that the association between microbiome and immune gene expression increases in 30 infected individuals, and varies with parasite genotype. In addition, we showed that S. solidus hosts 31 a complex endo-microbiome and that the abundance and prevalence of an unknown Chloroflexi in 32 the parasite correlate with expression of host immune genes including foxp3, tnfrl, cd97, stat6 and 33 marco.

34 Conclusions: Within this first comprehensive analysis of a cestode's interaction with bacteria, we demonstrate that (i) regardless of infection success, parasites contribute to modulating the host microbiome, (ii). when infection is successful, the host microbiome varies with parasite genotype 37 due to genotype-dependent variation in parasite immunomodulation, and (iii) the parasiteassociated microbiome is distinct from its host's and contribute to the host immune response to 39 infection. While a growing number of studies focus on determining the genetic and environmental 40 factors contributing to host microbiome composition, this study reveals that parasites, parasite 
41 genetic factors, and parasite microbiomes can contribute regardless of whether the infection was 42 successful. 


\section{Declarations}

\section{Ethics approval and consent to participate}

45 Work with fish was carried out in full accordance with laws governing use of animals for research

46 in Germany and care and well being of these animals was in compliance with EU legislation and

47 directives.

\section{Consent for publication}

49 Not applicable.

\section{Availability of Data and Materials}

51 All data is being made available through the NCBI database under BioSample accessions

52 SAMN08800217 to SAMN08800225, SAMN08805229 and SAMN08805231 for S. solidus field

53 samples and BioSample accessions SAMN08800337 to SAMN08800360, SAMN08805227,

54 SAMN08805228, SAMN08805230 and SAMN08805232 for G. aculeatus field samples. Cross

55 infection samples are available under the BioSample accessions SAMN16838912 to

56 SAMN16839059 for G. aculeatus samples and SAMN16839060 to SAMN16839129 for S. solidus

57 samples. Kit controls for the cross infection experiment can be found under accession numbers

58 SAMN16839130 to SAMN16839135. Code for all analyses is being made available through

59 Github at https://github.com/megan-hahn/stickleback-ssolidus-microbiome.

\section{Competing Interests}

61 The authors declare that they have no competing interests.

\section{$62 \quad$ Funding}

63 This project was supported by grants from the Eppley Foundation for Research and the Laurie

64 Landeau Foundation LLC awarded to NMD. This project is part of the Parasite Microbiome 65 Project. 


\section{Authors Contributions}

67 NMD designed the study. MH, AP, MK and NMD designed the experimental infection. MH, AP and MK collected fish and parasites and conducted the experimental infection. AP conducted the

69 RT-qPCR. MH executed the microbiome experiments and analyses. MH and NMD analyzed the

70 data with insight from FJ and RC. MH and NMD wrote the paper. All authors reviewed and

71 contributed to the final version of the manuscript.

\section{$72 \quad 3 \quad$ Introduction}

The past few years have been marked by the growing recognition that microbes contribute

74 actively to modulating the phenotype of their hosts, with applications in health, agriculture,

75 environmental sciences and evolutionary theory [1-5]. Studies have sought to determine a core

76 microbiome associated with a given host and to find the roles that these microbes play [6-8]. Others

77 investigate the impact of host genotype, diet, the environment, and diseases on the microbiome as

78 an attempt to infer consequences for the host [9-14]. But, how could two interacting organisms,

79 each with their own assemblage of microbes, impact each other's holobiont $[15,16]$ ? Parasitism

80 is one of the best examples of how species interactions are integrated and are ideal to start 81 answering this question.

The association between helminth infections and changes in the composition and diversity

83 of the host gut microbiota has been investigated in many systems [14, 17-20], in part as a mean to

84 better understand the link between parasite load, the microbiome, and inflammatory bowel

85 diseases [20-23]. Results differ greatly, with some studies showing an increase in microbial 86 diversity associated with infection [24-26], others showing a decrease in microbial diversity [13,

$8717,27,28]$, and some showing no changes $[18,29,30]$. Specific bacterial taxa were at times found 88 to be impacted such as Hymenolepis diminuta infection which is associated with a reduced 
abundance in Bacilli [17], Trichuris suis infection that is associated with an increased abundance

90 in Lactobacilli [13], and the liver fluke Opisthorchis viverrini that promotes Helicobacter growth

$91[14]$.

92 The differences that have been reported likely result from profound differences in host and

93 parasite eco-evolutionary history. Indeed, both host and parasite contribute to determining the

94 impact of infection on the gut epithelium, host immune response, or mucus production, all of which

95 could result in variations in host microbiota composition [31]. Profound differences between

96 parasite species exist depending on whether or not they reside in the gut and depending on their

97 impact on different host immune pathways [31]. Within a given host species, geographic variation

98 in parasite-mediated natural selection can result in geographic variation in immune response

99 mechanisms [32, 33]. Conversely, geographic variation in host immune response imposes

100 selection pressures on parasites that locally adapt to their hosts and display different virulence

101 mechanisms $[34,35]$. However, it remains to be determined whether this fine-tuned crosstalk

102 between host immune response and parasite virulence impacts host microbiota [36]. The finding

103 that a single parasite species can differentially impact the immune system and microbiota of

104 different host species [37], and conversely, that a single host species host different bacterial

105 communities depending on the parasite species it is infected with [38], support this hypothesis.

106 Disentangling whether the parasite itself impacts the host microbiome, or whether it is the

107 parasite interaction with the host immune system that leads in changes in microbiome composition

108 presents another challenge [39]. Parasites have been associated with direct modulation of their host

109 immune system via the production of virulence factors, breaching of host barrier surfaces such as

110 skin or intestinal epithelium, and production of excrements that interact with host immune cells

111 [40-45]. However, recent evidence has also shown that the host microbiome plays key roles in 
112 determining the host immune response [46, 47]. In particular, anti-inflammatory interleukins, 113 foxp3 and tgf- $\beta$ appear to be at the heart of the interplay between bacteria and the immune system $114[48,49]$. For example, the microbiome modulates the innate immune response of mice exposed to 115 influenza [50]. In this study, a microbiota-induced expression of IL-1 $\beta$ and IL-18 was found to be 116 associated with better outcome, and a distal inoculation of LPS to the colon was sufficient to 117 restore the immune response to influenza virus in the lung. Thus, to understand the impact a 118 parasite has on its host holobiont, it is essential to consider both immune function and microbiome 119 composition, and to investigate how they interact with each other.

120 Parasites can also host microbes and for some parasites, the role of bacterial symbionts in 121 virulence and pathogenesis has been thoroughly investigated. Many nematodes depend on 122 Wolbachia for normal development and fertility, and the bacteria also contributes to inflammation 123 and adverse reaction to anti-filarial drugs [51]. Similarly, the bacteria Neorickettsia has high 124 prevalence among digenean trematodes and is often transmitted to parasitized hosts causing 125 associated diseases [52-55]. But, for most parasite taxa, their associated microbiota remains 126 unknown, and in fact, the presence of other bacteria in trematodes and nematodes has not yet been 127 thoroughly investigated [56]. This might be because most helminths reside in the microbe-rich gut 128 of their hosts, and studies would have to disentangle the parasite microbiota from contaminants in 129 the sampled material [57]. Recent studies have shown that both nematodes and trematodes can 130 host a complex microbial community distinct from that of parasitized hosts and from the 131 environment, and evidence suggests that some of these bacteria might be vertically transmitted 132 [58-61]. However, the presence of bacteria in cestodes, the only taxa of helminths that do not have 133 a gut and absorb nutrients through the skin, has not yet been investigated. 
135 its threespine stickleback (Gasterosteus aculeatus) fish host using 16S amplicon rDNA 136 sequencing. This parasite system is particularly valuable because upon ingestion, S. solidus 137 egresses through the intestinal wall within 24 hours, which means that it is in direct contact with 138 the host gut microbiome for a very limited time [62]. The parasite then grows to sexual maturity 139 in the sterile peritoneal cavity of the fish which limits chances of contamination from the host 140 microbiota and allows a reliable assessment of the microbial communities associated with the 141 cestode. Thus, after an initial investigation of the microbiome of wild mature $S$. solidus and of 142 differences in the microbiome of infected and non-infected wild-caught sticklebacks, we 143 conducted experimental infections in control conditions. Threespine sticklebacks from two 144 different lakes in Alaska (Wolf and Walby lake), and from one lake in Germany (Lake Grosser 145 Ploner, GSP), were experimentally exposed, and infected with S. solidus from Wolf and Walby 146 lake in Alaska, and from one lake in Norway (Skogseidvatnet, SKO) (Figure 1). We tested the 147 effect of exposure to the parasite and infection on stickleback microbiome composition. Then, we 148 determined the role of host and parasite genetic factors in the effect of parasitism on the stickleback 149 microbiome. We also characterized S. solidus microbiome and tested whether the parasite 150 microbiome is shaped by environmental (the host microbiome) or genetic factors. Finally, using 151 stickleback genes involved in innate and adaptive immune responses, we identified genes whose 152 expression correlated with bacterial abundance in either the host or the parasite, and tested the 153 impact of infection, host genotype and parasite genotype, thus revealing immune pathways at the 154 crosstalk of $S$. solidus-bacteria interaction. 

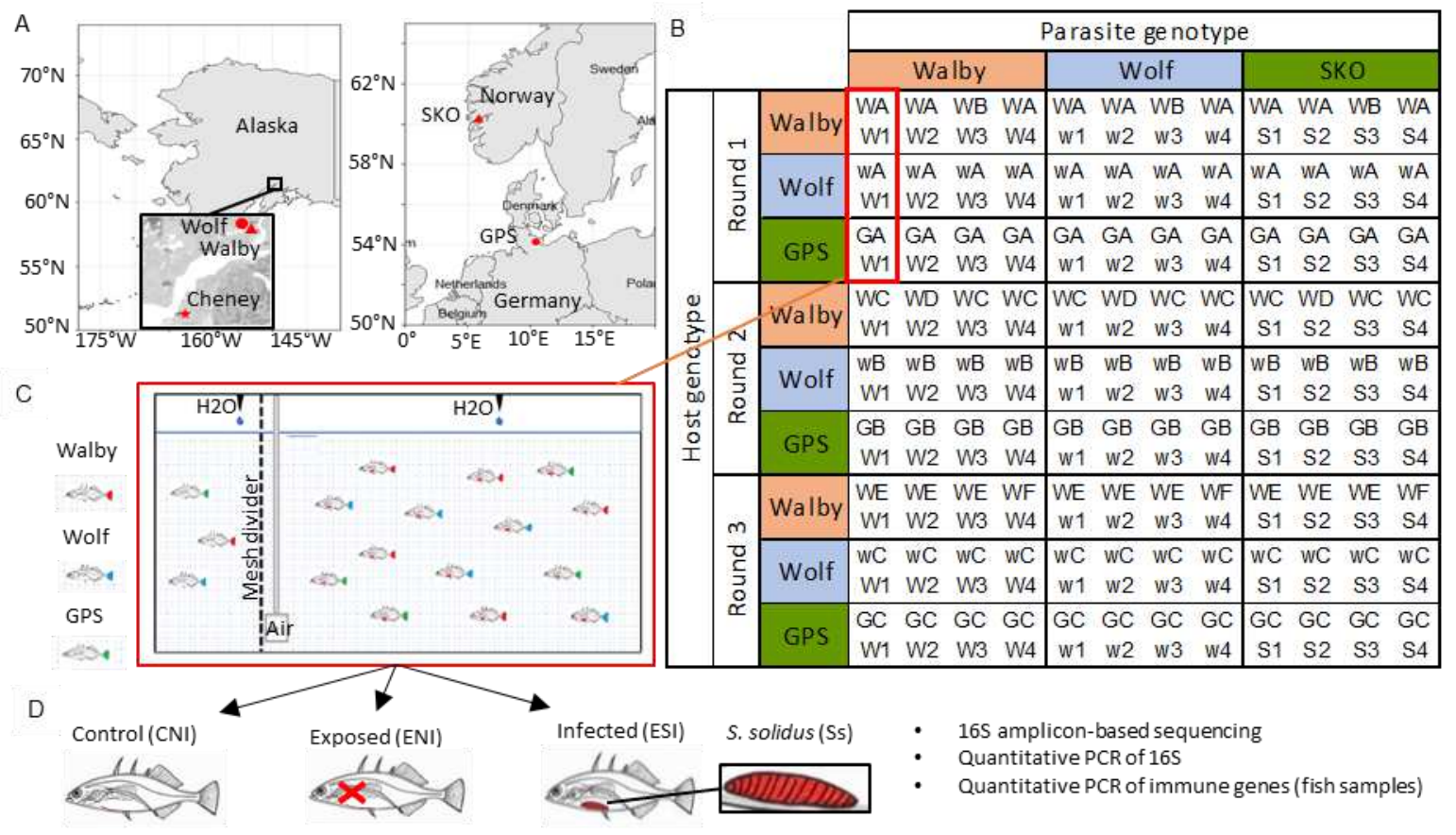

$\mathrm{H} 2 \mathrm{O} / \mathrm{H}$

$15^{\circ} \mathrm{E}$

Figure 1: Summary of experimental design. (A) The map displays all sampling locations. The

field study was conducted on sticklebacks and S. solidus from one location in Alaska (Cheney).

The three genotypes of stickleback hosts (Walby (W), Wolf (w) and GPS(G)) and three genotypes of S. solidus (Walby (W), Wolf (w) and SKO (S)) used for experimental infection were collected from lakes in Alaska and Europe. (B) Design matrix showing the organization of rounds and tanks based on fish genotype (denoted by letters) and parasite genotype (denoted by numbers). A total of 36 tanks were used. One example is squared in red. (C) Each of the tanks contained three sham control fish (left) from Wolf (Red Tail), Walby (Green Tail), and GPS (Blue Tail) separated by a mesh divider from 5 Wolf fish, 5 GPS fish, and 4 Walby fish that had been exposed to parasites (right). (D) Upon dissection, the success of infection was assessed, and exposed individuals were classified as exposed non-infected (ENI) or exposed successfully infected (ESI). All successfully infected fish were processed, and corresponding ENI and control non-infected fish from the same tanks and fish origin were processed as controls. 


\section{Results}

172

\section{The microbiome of wild-caught stickleback hosts and S. solidus parasites}

As an initial approach to investigate $S$. solidus interaction with microbes, we field caught threespine sticklebacks from Cheney lake, in Alaska to characterize and compare the microbiome of the gut of six non-infected fish, six infected fish, and corresponding nine S. solidus plerocercoids extracted from their body cavity. We used a quantitative PCR (qPCR) approach with two sets of universal primers targeting the bacteria $16 \mathrm{~S}$ rRNA gene as a proxy for bacterial load and $16 \mathrm{~S}$ amplicon-based sequencing to characterize the microbiome composition. Comparisons between non-infected and infected sticklebacks revealed profound differences in microbial abundance and diversity (Log Cell p<0.01; PCoA axes 1 and 2 unpaired T-test $\mathrm{p}<0.05$; PERMANOVA $=\mathrm{p}<0.05$; PERMDISP $=\mathrm{p}<0.05$; Figure S1 and S2). Sequencing of the surface microbiome of S. solidus, and of the body cavity of sticklebacks yielded results similar to negative controls (Figure S3). In contrast, S. solidus endomicrobiome was as diverse as the stickleback microbiome, but with distinct compositional differences (PCoA axis 1 paired T-test $\mathrm{p}<0.05$; PERMANOVA $=\mathrm{p}<0.05$, Figures S3-5). Actually, bacterial load and alpha diversity in S. solidus was not significantly different from bacterial load in non-infected fish ( $\mathrm{p}<0.05$, Figure S1-2 and S4). Confirming this result, the presence of cultivable bacteria in homogenates of plerocercoids plated on agar, but not on plerocercoid surface confirmed that the parasite harbors an endomicrobiome (Figure S6).

\section{Cross infection experiment}

Following experimental infections with hosts and parasites of different origin (Figure 1), we quantified and sequenced the $16 \mathrm{~S}$ genes of a total of 42 control non-infected sticklebacks (CNI), 35 exposed but non-infected sticklebacks (ENI), and 71 exposed and successfully infected 
193 sticklebacks (ESI), and corresponding S. solidus (Ss). Our results confirmed that S. solidus and G.

194 aculeatus harbor a distinct microbiome, and that exposure and infection alters the host microbiome 195 (Figure S7-8).

196 The microbiome of threespine sticklebacks varied with exposure, infection, host origin, 197 and parasite origin (Figure S9, Figure 2). Comparisons of the microbiome composition of CNI fish 198 revealed constitutive differences between Alaskan and European sticklebacks (Figure 2A). 199 Exposure to S. solidus was associated with small changes in beta diversity (Figure 2B), but resulted 200 in an increase in differences in microbiome diversity metrics among fish of all three origins (Figure 201 2C). Parasite origin played a less profound role and limited differences in diversity were found 202 between fish exposed to Walby and SKO parasites (Figure 2C). Successful infection with $S$. 203 solidus was associated with an increase in bacterial load that varied with parasite origin (Figure $2042 \mathrm{~B}$ and 2D). The microbiome of infected fish was dominated by more taxa than non-infected fish 205 (Figure 2B). Finally, parasite origin, but not host origin, was associated with differences in 206 microbiome composition among infected sticklebacks (Figure 2D), characterized by a distinct 207 clustering of fish infected by European and Alaskan parasites. Finally, regarding the microbiome 208 of S. solidus plerocercoids, parasite origin, but not fish origin was associated with subtle 209 differences in microbiome beta diversity (Figures 2E and S9). 

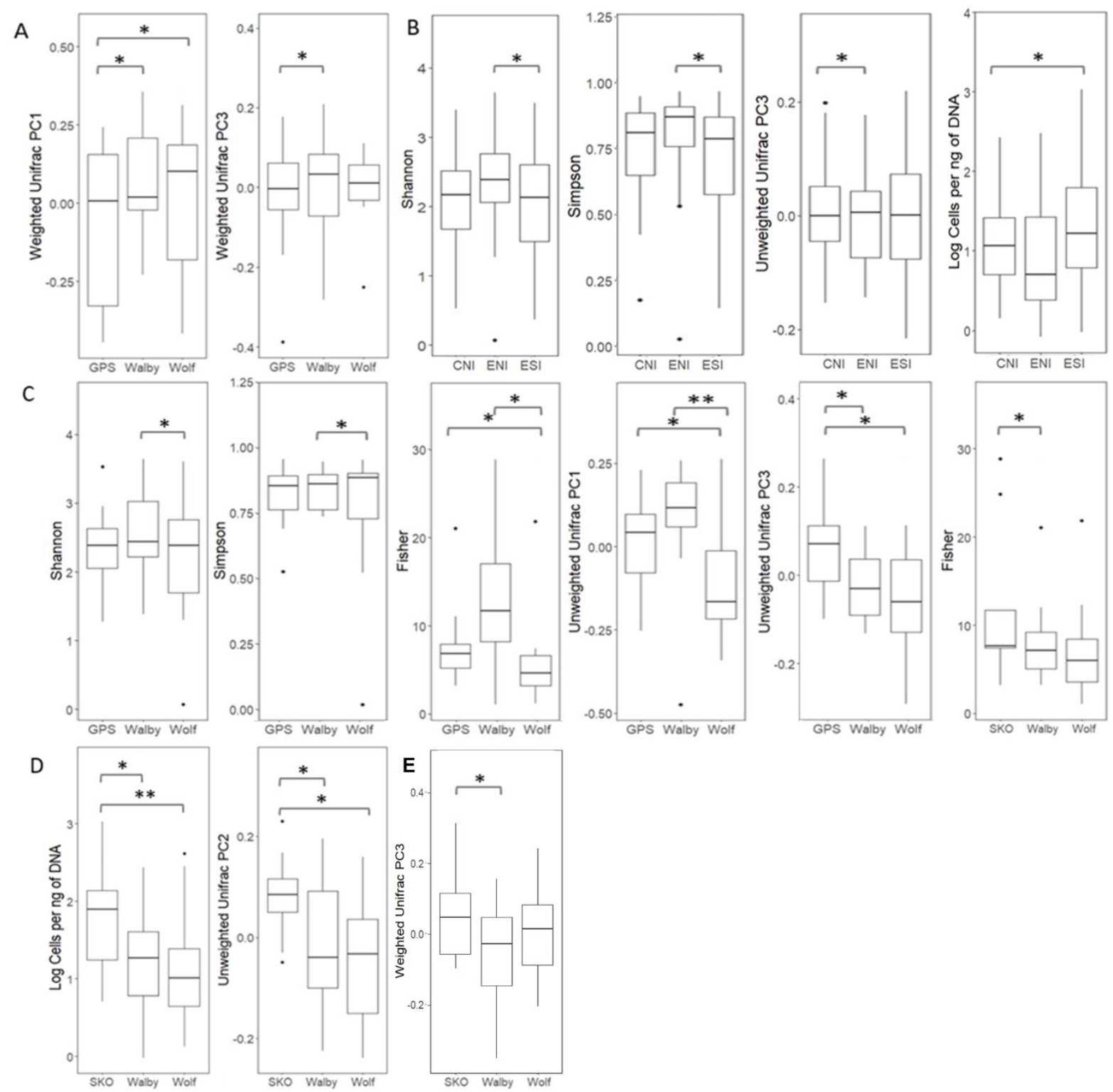

212 Figure 2: Stickleback bacterial community composition vary with exposure, infection status,

213 host genotype and parasite genotype as revealed by LMMs. (A) Among the control non-

214 infected fish, the microbiome composition varies with fish genotype. Differences were found

215 between Walby $(n=17)$ and GPS fish $(n=15)$ (Weighted Unifrac, Axes 1 LMM p=0.038; and Axis

216 3, Unweighted Unifrac, Axis 3, $\mathrm{p}=0.028)$ and between Wolf $(\mathrm{n}=10)$ and GPS fish (Unweighted

217 Unifrac Axis 1, p=0.024) . (B) Exposure and infection impact the microbial diversity and 
218 composition. Exposure impacts beta diversity (Weighted Unifrac, Axis 3, p=0.031), whereas

219 successful infection is associated with changes in bacterial load $(\mathrm{p}=0.01)$ and alpha diversity

220 (Shannon $\mathrm{p}=0.039$, Simpson $\mathrm{p}=0.013$ ). (C) Among exposed but non-infected fish, the microbiome

221 diversity and composition vary with host genotype and parasite genotype. Differences were found

222 between Wolf $(n=9)$ and GPS $(n=14)$ fish (Fisher $\mathrm{p}=0.008$, Unweighted Unifrac Axis $1 \mathrm{p}=0.005$,

223 and $3 \mathrm{p}=0.013)$, between Walby $(\mathrm{n}=10)$ and Wolf fish (Shannon $\mathrm{p}=0.034$, Simpson $\mathrm{p}=0.020$, Fisher

$224 \mathrm{p}=0.004$, Unweighted Unifrac Axis $1 \mathrm{p}=0.006$ ) and between Walby and GPS fish (Unweighted

225 Unifrac Axis $3 \mathrm{p}=0.041$ ). Differences were found between alpha diversity of fish exposed to Walby

226 and SKO parasites (Fisher $\mathrm{p}=0.018$ ). (D) Among infected sticklebacks, microbiome composition

227 varies with parasite origin and host-parasite co-evolutionary history. Fish infected with SKO

$228(\mathrm{n}=18)$ parasites had higher bacterial load than fish infected with Walby $(\mathrm{n}=27, \mathrm{p}=0.010)$ and Wolf

229 parasites $(\mathrm{n}=25, \mathrm{p}=0.001)$. Unweighted Unifrac distance revealed distinct clustering of fish

230 infected with SKO parasites and fish infected with Walby or Wolf parasites (Axis 2, p=0.046 and

$231 \mathrm{p}=0.047$ respectively). (E) Within parasites, microbiome composition varies with parasite origin.

232 Weighted Unifrac distance revealed distinct clustering of the microbiome of SKO $(\mathrm{n}=18)$ and

233 Walby $(\mathrm{n}=27)$ parasites (Axis $3, \mathrm{p}=0.035)$. The bottom and top edges of the boxes mark the $25^{\text {th }}$

234 and $75^{\text {th }}$ percentiles. The horizontal line denotes the median. Whiskers mark the range of the data

235 excluding outliers (dots).

236

237 Taxa distribution

We observed 458 bacterial families associated with G. aculeatus samples and 216 families

associated with S. solidus. Of these, 202 families were found in both fish and parasites. The 253 
241 prevalence, contributing a cumulative relative abundance of $6.9 \%( \pm \mathrm{SE} 0.02)$ and $0.1 \%( \pm \mathrm{SE}$

242 0.001), respectively. Thus, all abundant bacterial families in S. solidus were found in sticklebacks

243 (Figure 3A). But, the most abundant and most prevalent bacteria in parasites and fish are not the 244 same and comparison of the relative abundance of bacterial taxa between individual S. solidus and 245 their corresponding fish host revealed an absence of relationship (Figure 3A, Figure S8). In total, $24693 \%$ of the ASVs, and 9.4\% of the families present in ESI sticklebacks were never found in $S$. 247 solidus, including the Vibrionaceae that represented an average relative abundance of 5.5\% in ESI 248 fish. Most of the abundant ASVs in S. solidus had low abundance and prevalence in sticklebacks, 249 including ASVs of Bacillaceae, Lactobacillus, Chloroflexi, Rhodospirillales, Actinomycetales, 250 and Chitinophagaceae (Figure 3B). Actually, the family Bacillaceae was the most abundant in 251 both fish and parasites (with a relative abundance of $30.6 \%$ and $7.5 \%$, respectively, Figure $3 \mathrm{~A}$ ), 252 but comparisons at the ASV level revealed major compositional differences. Of the 144 ASVs that 253 compose this family, only 16 were found at least once in both S. solidus and G. aculeatus, and all 25412 most abundant and prevalent ASVs of Bacillaceae were significantly differentially abundant 255 (DESeq Adj p <0.05) in fish and parasite: four are more abundant in parasites and eight are more 256 abundant in fish. A neighbor joining analysis of all ASVs of Bacillaceae revealed that those most 257 prevalent and abundant in S. solidus cluster separately from those most prevalent and most 258 abundant in G. aculeatus (Figure S10). 

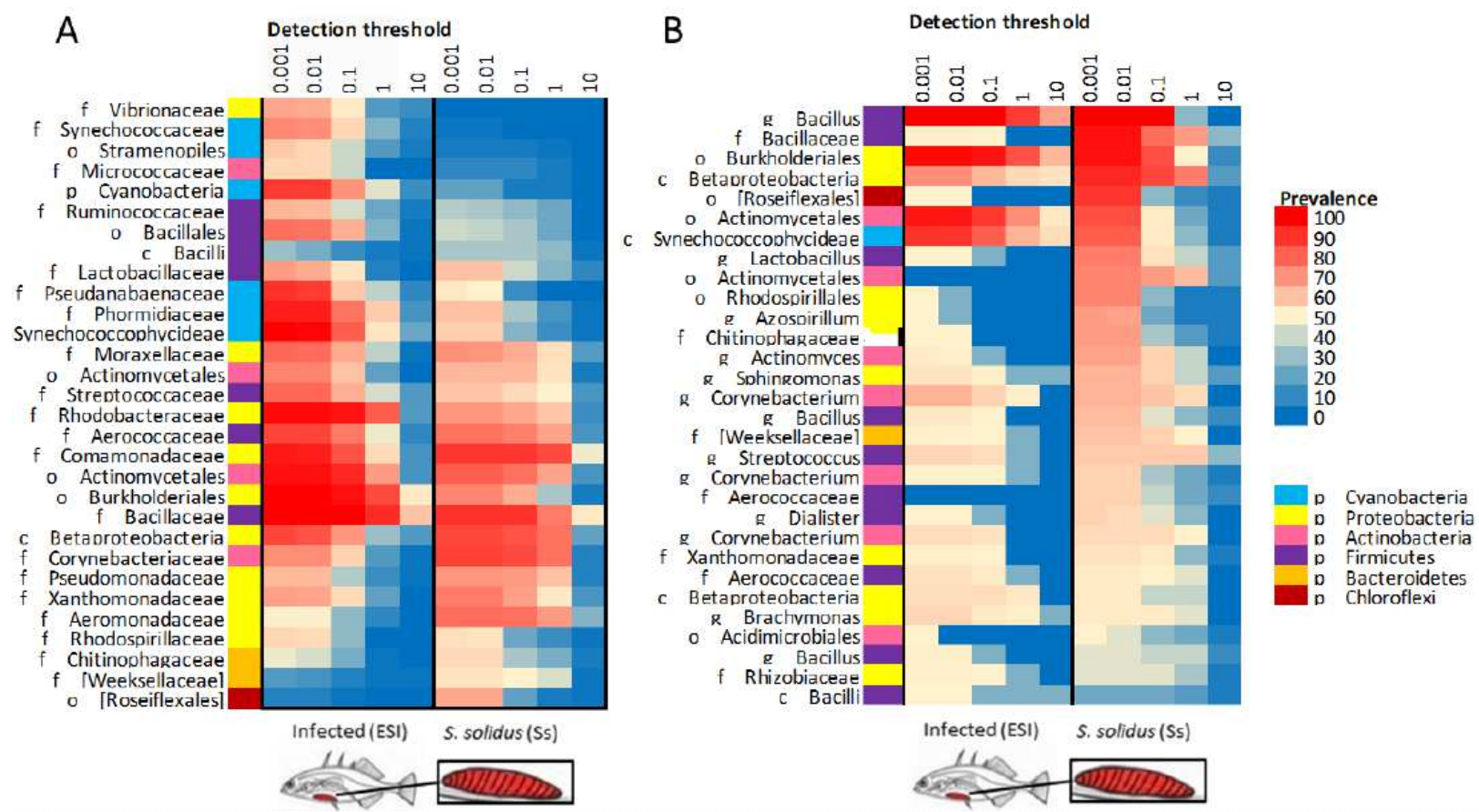

Figure 3: Bacterial taxa at multiple taxonomic levels vary in relative abundance and (defined in terms of minimum relative abundance) for successfully infected (ESI) sticklebacks, and corresponding S. solidus. A/ 30 most abundant bacterial families across all samples. B/ 30 most abundant ASVs in S. solidus. The phylum is provided as a color code next to the taxa name. associated with higher relative abundance of Rhodobacteraceae, and with a lower relative abundance of Betaproteobacteria, whereas successful infection resulted in a higher relative

271 abundance in Vibrionaceae, an unknown Synechococcophycideae and another unknown 272 Cyanobacteria. 
274 of bacterial families (Figure S11). In CNI, host genotype was associated with variation in 275 Streptococcaceae, Phormidiaceae and Ruminococcaceae. Focusing on ENI, the relative 276 abundance of an unknown Chloroflexi varied with both parasite origin and fish origin, and the 277 relative abundance in the aforementioned Rhodobacteraceae (impacted by exposure to S. solidus) 278 varied with parasite origin. In ESI, two Synechococcophycideae (family Synechococcaceae and an 279 unknown family) varied in relative abundance depending on fish origin. Similarly, two families of 280 Bacillales (Bacillaceae and an unknown family) and two families of Betaproteobacteria (an 281 unknown Burkholderiales and another unknown family) in addition to the family 282 Pseudanabaenaceae (Synechococcophycideae) varied in relative abundance depending on parasite 283 origin.

In $S$. solidus, the relative abundance in bacterial families varied with the origin of the 285 infected host and with parasite origin. Of interest, 11 families were found to vary in abundance 286 with both host and parasite origin, including the aforementioned unknown family of Bacillales. In 287 addition, the relative abundance in Lactobacillaceae varied with parasite origin, whereas the 288 relative abundance in Phormidiaceae and Rhodospirillaceae varied with fish origin.

Impact of S. solidus on the interaction between immune gene expression and the microbiota

292 gene expression and the microbiome (Figure S12, Figure 4). Spearman's rank correlation revealed 293 an interaction between gene expression profiles and beta diversity metrics (Unweighted unifrac 294 distance PCoA axis $2 \mathrm{p}=0.0288$ and PCoA axis $3 \mathrm{p}=0.0017$; Figure 4A). The relationship between 295 microbiome composition and gene expression profile was more pronounced in infected 
296 individuals, the greater slope indicating that the parasite increases the dependency between 297 immune response and microbiome composition (Figure 4B). Among infected individuals, parasite 298 origin, but not host origin was associated with significant differences in the strength of the 299 correlation between immune gene expression and microbiome composition (Figure 4B). These 300 correlations appear to be driven by a subset of bacterial families, among which some were 301 positively correlated with gene expression whereas others were negatively correlated (Figure 4C). 302 The most significant correlations involved Treg-inducing genes stat4, stat6 and ill6, Treg 303 associated gene $f o x p 3$, complement factor $c f b$, anti-microbial innate regulatory genes $c d 97$ and 304 marco, and the regulator of inflammation $\operatorname{tnfr} 1$ (Figure 4C, Figure S12). More specifically, the 305 relative abundance of Synechococcophycideae, Lactobacillaceae and Pseudanabaenaceae were 306 negatively correlated with immune gene expression (Figure 4E-G) whereas the relative abundance 307 in Vibrionaceae was negatively correlated with the expression of the immune response genes 308 (Figure 4H). Relative abundance in a different subset of bacteria, namely Aerococcaceae, 309 Streptococcaceae and an unknown Actyinomycetales were positively correlated with a higher 310 hepatosomatic index (Figure 4D). 


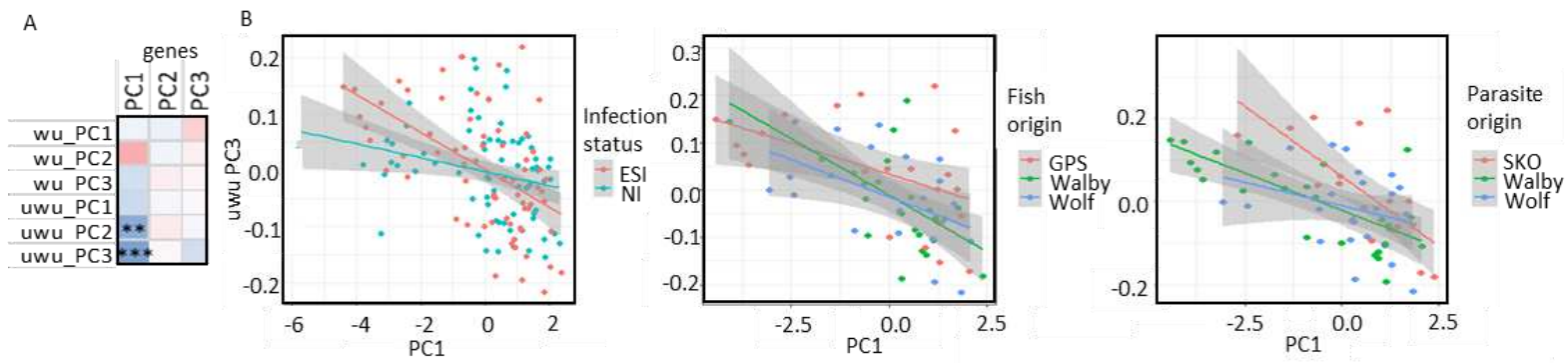

C Immune response genes phenotype

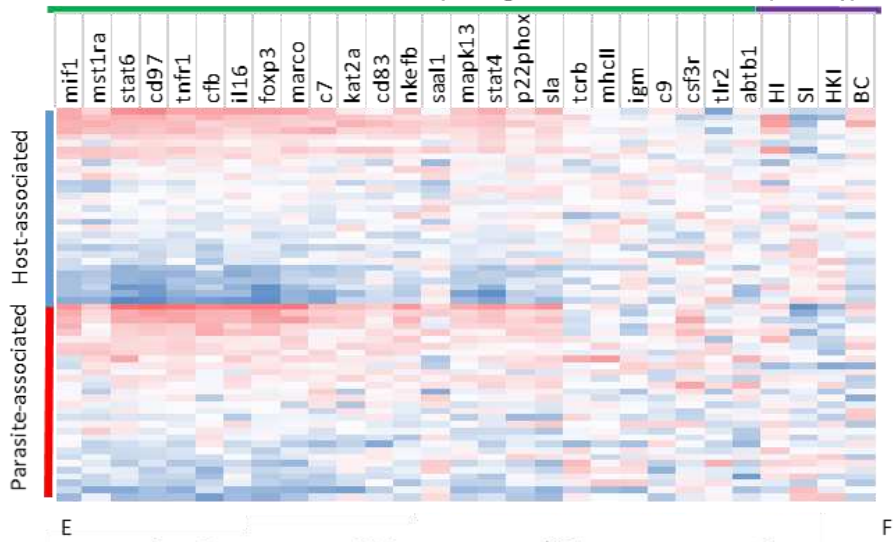
D 10
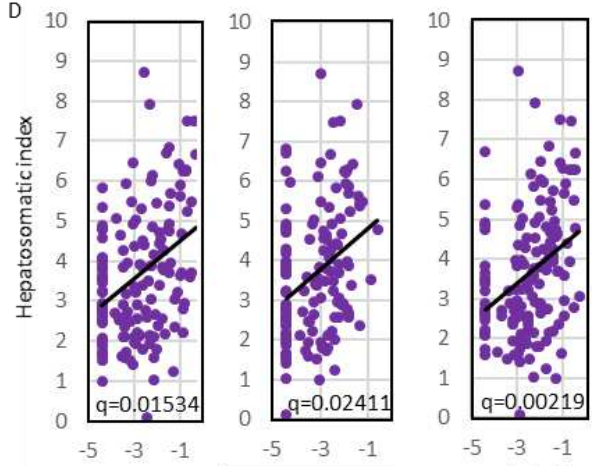

Log10(rel ab) in Log10(relab) in $\log 10(\mathrm{rel} a b)$ in f_Streptococcaceae o_Actynomicetales f_Aerococcaceae
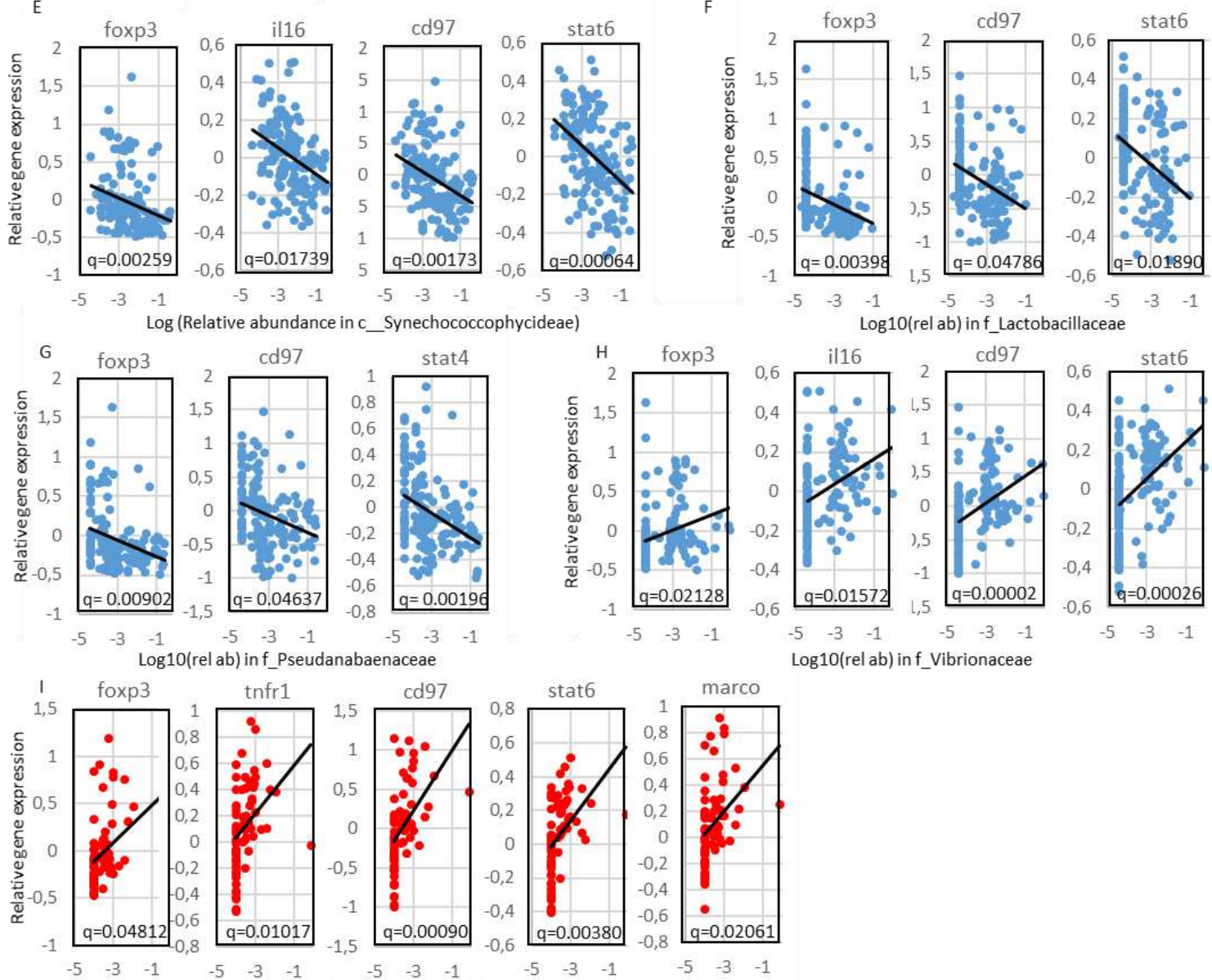

Log10(rel ab) in c_Chloroflexi 
312 Figure 4: The host phenotype depends on the composition of the microbiome of the host and

313 of its parasite. A. Correlation heatmap depicting gene expression - microbial profile correlations.

314 Color of the squares indicate the strength of Spearman's rank correlation. Asterisks indicate

315 significance of the correlation $(* \mathrm{q}<0.05 ; * * \mathrm{q}<0.05 ; * * * \mathrm{q}<0.001)$. B. Comparison of correlations

316 between gene PC1 and uwuPC3 depending on infection status, host genotype and parasite

317 genotype. Significant differences are observed when the confidence interval at the lower and/or

318 higher values do not overlap with the mean of the other one. C. A. Correlation heatmap between

319 the expression of genes, and phenotypic indices (HI, Hepatosomatic index; SI, Splenosomatic

320 index, HKI Head-kidney index; BC, Body condition) and the relative abundance in the most

321 abundant acterial families in the host G. aculeatus, and its parasite $S$. solidus. Color indicate the

322 strength of Spearman's rank correlation. The complete figure with indication of significance is

323 available in Figure S12. Significant Spearman's rank correlation (with FDR <0.05) are plotted in

324 (D) between the relative abundance in Streptococcaceae, an unknown Actynomicetales and

325 Aerococcaceae in the host with Heptaosomatic index $(\mathrm{N}=145)$, in $(\mathrm{E})$ between the relative

326 abundance of Synechoccophycidae in fish and the relative expression of foxp3, ill6, cd97 and stat6

$327(\mathrm{~N}=145)$, in $(\mathrm{F})$ between the relative abundance in Lactobacillaceae in fish and the relative 328 expression of foxp3, cd97 and stat6 $(\mathrm{N}=145)$, in $(G)$ between the relative abundance in 329 Pseudanabaenaceae in fish and the relative expression of foxp3, cd97 and stat4 $(\mathrm{N}=145)$, in $(\mathrm{H})$ 330 between the relative abundance of Vibrionaceae in fish and relative expression of of foxp3, il16, 331 cd97 and stat6 $(\mathrm{N}=145)$ and in (I) between the relative abundance of Chloroflexi in the parasite 332 and relative abundance in foxp3, tnfr 1, cd97, stat6, and $\operatorname{marco}(\mathrm{N}=69)$. The significant Spearman 333 correlation, after Bonferroni correction, are provided on each plot. 
The putative role of $S$. solidus-associated bacteria on immune gene expression

Finally, we investigated whether bacterial families associated with S. solidus correlated with immune gene expression in infected individuals. Our analysis revealed a positive correlation between some of the highly prevalent bacterial families and immune gene expression in infected fish (Figure $4 \mathrm{C}$ ). Most specifically, the relative abundance of the family of Chloroflexi in $S$. solidus was correlated with a significantly lower PC1 values, and higher expression of stat6, foxp3, tnfrl, $c d 97$, and marco demonstrating the impact of parasite-associated bacteria on host immune response to infection (Figure 4I).

\section{$5 \quad$ Discussion}

\section{S. solidus hosts a diverse microbiome}

Our results provide the first set of evidence of an endomicrobiome in the cestode S. solidus. We collected $S$. solidus plerocercoids from the body cavity of $G$. aculeatus, so that the parasite was no longer in contact with the host gut microbiota, limiting the potential for contamination [76].

We did not culture any bacteria after spreading freshly sampled plerocercoids on agar suggesting the absence of a surface microbiome. Using 16 sequencing, we further tested the presence of bacteria in the body cavity of parasitized fish and on the surface of $S$. solidus, and obtained results similar to blank controls, confirming that the fish body cavity is sterile, and that no bacteria are present on the cestode tegument. In contrast, we could grow colonies on agar and characterized a complex microbiome from plerocercoid homogenates. In both field and laboratory experiments, the microbiome of S. solidus was dominated by Proteobacteria and Firmicutes, and to a lesser extent with Bacteroidetes, Actinobacteria and Chloroflexi. The microbiome of the trematodes

Coitocaecum parvum and Opisthorchis viverrini are also dominated by Firmicutes, Proteobacteria 
and Actinobacteria $[14,60,63,64]$. Together, these results strongly support the hypothesis that any parasitic flatworm may host a microbiome and encourage studies to investigate the structure and function of helminth microbiome.

Our results show that $S$. solidus microbiome is not a random assemblage of microbes present in the parasite environment and suggest that host-based selective forces play a role in shaping the parasite microbiome composition and diversity. Microbial community assembly can consist of vertical transmission of symbiotic members, and horizontal acquisition of microbes from the organism's environment (through random process or host-based selection). Vertical transmission and host-based selection of environmental microbes can lead to diversification of microbial assemblages, and even phylosymbiosis, where the clustering of microbiome composition data concur with the phylogeny of the hosts [65]. In accordance, we found significant differences in our two Alaskan S. solidus populations, and the European population, that are all recognized as genetically distinct lineages $[66,67]$. But, our results indicate that the S. solidus microbiome is markedly different from its host microbiome, even at the ASV level: different ASVs had high prevalence and relative abundance in parasites and fish. The presence in S. solidus of ASVs that were absent in threespine sticklebacks suggest that some of these bacteria could be vertically transmitted. In disagreement with this hypothesis, none of the ASVs found in S. solidus had high prevalence in parasites of only one origin. Yet, ASVs of Bacillaceae of S. solidus tend to cluster together and separately from ASVs of Bacillaceae associated with G. aculeatus. We suggest that $S$. solidus might select bacteria from its environment at previous stages of its life cycle. Given the diversity of microbial reservoirs from which this parasite may acquire microbiota throughout its complex lifecycle (definitive bird host, environmental water and soil, intermediate copepod host and intermediate stickleback host), more studies need to be completed to determine 
381 the sources for the parasite microbiome, the mechanisms of acquisition, and the conditions that

382 impact the maintenance of these microbial communities.

Both exposure and infection influence the host microbiome composition

We observed an impact of both exposure and infection by S. solidus on the fish gut microbiome even though the parasite had not been in contact with host-associated microbes for weeks at the time of sampling. Regardless of host or parasite genotype, and regardless of the lab environment, exposure and infection by S. solidus were associated with consistent changes in the microbiome. Indeed, herein, and within Ling et al. [36], a significant increase in Rhodobacteriales was found associated with exposure and infection by S. solidus. In addition, both our field study and experimental infections revealed a previously unreported increase in alpha diversity metrics and bacterial load in infected individuals. We also observed variations among studies that may be 392 due to differences in environmental conditions, of using sticklebacks with different genetic 393 background, and from using different strains of parasites. Indeed, while Ling et al [36] showed an 394 increase in Lactobacilliales in infected individuals, we observed an increase in Vibrionales and 395 Desulfovibrionales following experimental infection and field sampling, respectively. The 396 colonization of the gut of infected fish by these bacteria may be dependent on their initial presence 397 in the environment of the fish. Unfortunately, this is a limitation of our design, as we did not 398 characterize the environmental water microbiome, which prevented us from testing the hypothesis 399 that these bacteria had variable presence in these different environmental and field settings. All 400 these bacterial families are of interest because of their pathogenic potential [68-70], and might 401 indicate that infection with S. solidus increases G. aculeatus susceptibility to pathobionts as 402 previously reported for other helminths [71]. 
The impact of exposure to $S$. solidus on the microbiome is intriguing because there is

404

405

406

407

408

409

410

411

412

413

414

415

416

417

418

419

420

421

422

423

424

425

currently no evidence that exposure alone induces a sustained effect on G. aculeatus. In fact, until now, studies comparing threespine stickleback immune gene expression and other immunological parameters could not reveal differences between control and exposed but non-infected sticklebacks $[33,72]$. However, we recently observed an impact of exposure on threespine stickleback hepatosomatic index and body condition, traditionally used as a measure of host condition [33, 73]. This reduced host condition upon exposure suggests that the cost of resistance to infection, and elimination of the parasite through cell-mediated immunity within the first two weeks of infection [74] could indirectly impact the microbiome of exposed sticklebacks.

\section{The interaction of host- and parasite-associated bacteria with the host immune system}

As an initial approach to characterize the underlying mechanisms, for the indirect effect of S. solidus presence in the body cavity on the host microbiome, we looked for the impact of exposure and infection on the correlation between immune gene expression and microbiome composition. The most significant correlations involved foxp3, a specific markers for Treg and

Treg-inducing genes stat4, stat6 and il16, the $c f b$ central to the complement system, antigen $c d 97$ that promote cell migration to sites of inflammation, the scavenger receptor cysteine-rich protein marco that binds bacteria, and the regulator of inflammation $\operatorname{tnfrl}$ that activate the transcription factor NF-kappaB. Other genes involved in innate immunity, and the Th1 or Th2 adaptive immune response did not correlate with bacterial abundance, despite being strongly modulated following S. solidus infection [56]. Correlation between immune parameters and gut microbes are well documented in other species including horses [75], mice [76-78], rats [79] and humans [80, 81] and have been used to infer pathways involved in the interaction. In particular, commensal bacteria are associated with an anti-inflammatory regulatory $\mathrm{T}$ cells, whereas pathogens induce a pro- 
426 inflammatory response. We found a negative correlation between pro-inflammatory immune gene 427 foxp3, ill6, stat4, stat6 and cd97 and the family Lactobacillaceae, and the Cyanobacteria 428 Synechococcophycidae and Pseudanabaenaceae but a positive correlation of the same genes with 429 Vibrionaceae, indicating that these bacterial families associated with G. aculeatus are likely 430 commensals and pathogens respectively. The strength of the interaction between microbiome 431 composition and gene expression profile was higher in infected individuals, which is explained by 432 the higher relative abundance in Vibrionaceae and Synechoccophycideae in infected individuals.

433 This results indicates that $S$. solidus reinforce the immune communication between the host and 434 its microbiome. prevalent in S. solidus and expression of pro-inflammatory genes foxp3, tnfrl, cd97, stat6 and

437 marco. Although not significant with our sample size, other bacteria associated with S. solidus, 438 such as two families of Rhodospirillales and a Chitinophagaceae, also correlate with a pathogen439 like immune gene expression pattern. Even though little is known about these bacteria, Chloroflexi 440 and Rhodospirillaceae include opportunistic pathogens [82, 83]. Helminths, including S. solidus 441 induce a Treg response, ensuring their persistence during chronic infection, and preventing a 442 pathological inflammatory response that would be detrimental to the host $[72,75,84]$. Thus, the 443 observed parasite-associated bacteria could contribute, or hinder the mechanisms of parasite 444 modulation of host immune response. While it has previously been shown that host associated 445 microbiota can contribute to host-parasite coevolution [85], this study demonstrates the necessity 446 of investigating the role of parasite-associated microbes.

447 A host-genotype dependent constitutive response to exposure impacts the microbiome 

depends on host genotype. In control individuals, we observed host genotype-specific constitutive differences in microbiome composition that confirm that host-based selective processes shape the organism microbiota [86-88], probably due to constitutive differences in immune gene expression

452 pathways between populations $[33,73,89,90]$. In agreement, we found host-genotype dependent 453 differences in the relationship between key immune genes and abundance of bacterial families, 454 indicating that fish of different origins have developed different sensitivity to different bacterial 455 families. Interestingly, we found that exposure to S. solidus increased differences based on host 456 genotypes. In contrast, limited differences between fish populations were found after infection by S. solidus. The same observation was recently made concerning the immune system of threespine 458 sticklebacks exposed to $S$. solidus: constitutive differences in immune gene expression between 459 populations increased upon exposure to $S$. solidus, whereas gene expression profiles converged 460 upon infection [33]. These results indicate that a non-specific, but a host genotype dependent 461 response to $S$. solidus modulates the Threespine stickleback microbiome composition in exposed 462 individuals. In infected individuals, the decrease variance is consistent with parasite manipulation of host phenotype, homogenizing responses towards an optimum that favors parasite fitness [91].

\section{The impact of infection on the host microbiome is parasite genotype dependent} is physically separated from the gut microbiome - can nevertheless impact its host gut microbiome

467 composition, we showed that genetic variations in parasitic worms influences the ways the

468 microbiota changes. This parasite-genotype effect was observed in all exposed threespine 469 sticklebacks, but it was stronger in successfully infected fish. Related species of helminth parasites 470 have been shown to modulate their host microbiomes differently as is the case with the cestodes 
471 Hymenolepis dimunita and Hymenolepis nana [38], and with the nematodes Trichuris suis and

472 Trichuris muris $[12,13]$. However, our study is the first to demonstrate that different genotypes of

473 the same parasite species have different impacts on host microbiome composition and diversity.

474 The observed limited parasite genotype effect on the microbiome of fish that resisted the parasite,

475 together with the fact that the parasite has a very limited time to directly interact with gut microbes

476 supports the hypothesis of a differential modulation of the immune response by different parasite

477 genotypes that indirectly impact the host microbiome. Variations in the immune response the host

478 mount during infection can either result from locally adapted parasites that differentially

479 manipulate the host immune system [90, 92], or from the host mounting a defense mechanism that

480 differentiate between parasite genotypes [33, 73, 93, 94]. In support of parasite local adaptation,

481 we showed that parasites of different origin differentially impact the correlation between host

482 microbiome composition and immune gene expression. Our results suggest that variations in the

483 microbiome of infected hosts vary with the mechanisms of local host-parasite counter adaptation

484 due to the tight association between microbiome composition and immune gene expression.

485 Conclusions and perspectives

Tapeworms represent an important problem for health authorities with over 40 species

487 infecting humans as definitive hosts, and 15 during the larval stage, many of which are included

488 in the list of neglected zoonotic diseases by the World Health Organization due to their impact on

489 human health and morbidity in impoverished nations [95]. But helminths in general are also under

490 study because of their beneficial regulatory role in autoimmune diseases, and inflammatory

491 diseases including atopic dermatitis, inflammatory bowel diseases, asthma and allergies.

492 Helminthes typically induce a Th2 and Treg immune response. There is a growing recognition of

493 the role of microbes in helminths ability to modulate the host immune system and of the imperative 
494 to study host-parasite-microbe interactions $[96,97]$. Herein, we showed the presence of a complex 495 microbiome within S. solidus and provided evidence for non-random selection of microbes from 496 the parasite environment. We also provide evidence that some of the parasite-associated bacteria 497 contribute to modulating the host immune system. Recently, we also discovered that viruses are 498 associated with $S$. solidus and that some of these are transmitted to parasitized hosts, which means 499 that viruses could also impact the host immune response [98]. Further studies are needed to test 500 whether S. solidus relies on these microbes for infection, or whether they are detrimental for the 501 parasite. Herein, we also demonstrate that the host microbiome, which varies with host genotype 502 in healthy individuals, is impacted by infection and depends on the parasite genotype in infected 503 individuals. The relative abundance of key members of the microbiome correlated with expression 504 of Treg, complement factor, and innate gene involved in recruiting cells to the site of inflammation, 505 and was impacted by infection status and parasite origin. This result suggest that infection by 506 different parasites differentially impact the immune communication between the host and its 507 microbiome, and that constitutive microbiome differences among hosts do not contribute to 508 differences in immune responsiveness to parasite infection. Further studies need to be conducted 509 to assess the role of host-associated and parasite-associated microbes in parasite fitness, and to 510 determine if microbes could be leveraged for developing new treatment strategies [99].

\section{Methods}

\section{$512 \quad$ Ethics statements}

The sampling was conducted under Fish sampling permit \#SF2015-263 and \#P17-025 and

514 fish transport permits \#15A-0084 and 17A-0024 provided by the State of Alaska Department of 515 Fish and Game to Michael Bell. Animal sampling and experiments in Germany and Norway were 516 approved by the Ministry of Energy Transition, Agriculture, Environment and Rural Areas of 
517 Schleswig-Holstein under reference number V 312-7224.123-34. All experiments were performed

518 in accordance with relevant guidelines and regulations in the Public Health Service Policy (PHS)

519 on Humane Care and Use of Laboratory Animals.

$520 \quad$ Processing of field collected specimens

521 In September 2015, threespine stickleback (Gasterosteus aculeatus) were collected from

522 Cheney Lake in Anchorage Alaska $\left(61^{\circ} 12^{\prime} 17^{\prime \prime} \mathrm{N}, 149^{\circ} 45^{\prime} 33^{\prime \prime} \mathrm{W}\right)$ to conduct an initial field

523 study. Fish were caught using un-baited minnow traps placed along the north shoreline of the lake

524 between 0.25 and $2 \mathrm{~m}$ deep, and at least $2 \mathrm{~m}$ apart. Fish were shipped to Stony Brook University,

525 kept in 20 -gallon tanks of $6 \%$ seawater at $5^{\circ} \mathrm{C}$ at the Flax Pond Marine Lab, and fed mysid shrimp

526 twice per day. Following an acclimation period of 3 weeks, and 12 hours of starvation, fish were

527 anaesthetized and euthanized in tricaine methane sulphonate solution (MS222), decapitated, and

528 dissected in sterile conditions. Prior to and in between dissections all surfaces were cleaned with

$52980 \%$ ethanol and betadine to remove contaminants. Before dissection, fish were brushed with

530 betadine to prevent contamination of the body cavity with microbes from the surface of the fish.

531 For dissection, an incision was made along the lateral line of the fish body, around the bony pelvis.

532 The cut extended from the pectoral fins to just anterior of the anus to avoid cutting the intestine.

533 The sex of the fish was assessed by visual inspection of gonads at the time of dissection and then

534 confirmed using PCR with sex specific primers as described in [100]. The presence of S. solidus

535 was recorded, and stomach and intestines were flash frozen in liquid nitrogen for future DNA

536 extraction. Swab samples of the body cavity of two non-infected and two infected individuals were

537 also collected. Two parasites were also vigorously shaken in sterile phosphate buffered saline

538 (PBS) and the resulting PBS was stored. These swab and PBS samples were used as confirmation 
that any bacteria found in $S$. solidus were indeed part of an endomicrobiome and not contamination from the fish body cavity or surface of the parasite. All samples were stored at $-80^{\circ} \mathrm{C}$ until use.

In Spring of 2016, threespine sticklebacks were caught, as described above, from Wolf lake

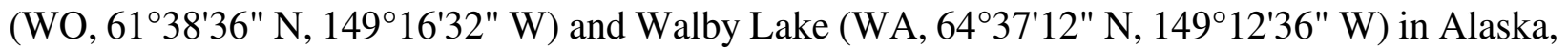

544 Grosser Plöner See (GPS, 549'16"N, 10²5'14"E) in Germany, and Lake Skogseidvatnet (SKO, 545 $\left.60^{\circ} 14^{\prime} 44^{\prime \prime} \mathrm{N}, 5^{\circ} 55^{\prime} 03^{\prime \prime} \mathrm{E}\right)$ in Norway in preparation for the cross infection experiment.

546 For Wolf, Walby and GPS, G. aculeatus eggs were dissected out of gravid females euthanized in MS-222. Eggs were fertilized with sperm suspension collected from euthanized male sticklebacks and then washed with acriflavine $(50 \mu \mathrm{L} ; 30 \mathrm{sec})$, methylene blue $(500 \mu \mathrm{L}$ per L from stock: $1 \mathrm{~g} / \mathrm{L}$ methylene blue; $30 \mathrm{sec}$ ), and 3ppt artificial seawater. Eggs were kept at $4{ }^{\circ} \mathrm{C}$ and shipped to the Max Planck Institute for Evolutionary Biology (MPI), Plon, Germany. Fin clips were taken from

551 the parents of each family to enable microsatellite analyses post experiment. Upon maturation, fish 552 were kept in a flow through system and fed ad libitum bloodworms every other day.

553 Mature $S$. solidus plerocercoids from Wolf, Walby and SKO were shipped to the MPI in freshly 554 caught conspicuously parasitized fish that were dissected immediately upon arrival. Pairs of $S$. 555 solidus plerocercoids were weight-matched and bred in vitro [101]. The eggs were then kept at $556 \quad 4^{\circ} \mathrm{C}$ in the dark until use.

\section{Cross infection experiment}

We conducted experimental infections of threespine sticklebacks with $S$. solidus in the 559 spring of 2017. Briefly, for each locality, three families of fish and four families of parasites were used (Wolf, Walby, and GPS for fish and Wolf, Walby and SKO for parasites). Macrocyclops 561 albidus copepods from a laboratory stock were singly infected with S. solidus procercoids as 
562 previously described [102]. Fish were starved for 24 hours before being fed either one singly

563 infected copepod or one non-exposed copepod (sham control). After 2 days, fish were transferred

564 into 16L aquaria. Fish exposed to a given parasite family were held together in the same tank. Each

565 tank held five exposed fish from Wolf, five exposed fish from GPS and four exposed fish from

566 Walby, in addition to one control fish per fish population (17 fish/tank). The common garden

567 design aims at controlling for tank effects observed in flow through systems (Figure 2) [103, 104].

568 Fish were fed with frozen Chironomids larvae three times a week. Due to the large number of

569 tanks used, we conducted three rounds of experimental infection. Each round, different families of

570 fish, and different families of parasites from each of the three populations were used.

571 Nine weeks after exposure, fish were euthanized with MS-222 and had morphometric data

572 recorded including fish standard length, fish total length, and fish weight. Upon dissection, masses

573 of the spleen, liver, and head kidney were weighed, and these tissues were preserved in RNAlater

574 (Thermofisher CAT\# AM7020). Intestine and plerocercoid (from successfully infected fish)

575 samples were flash frozen in liquid nitrogen for DNA extraction. A fin clip was collected and

576 preserved in ethanol to be used for microsatellite analysis and to determine fish origin. DNA was

577 extracted from fin clips collected from parents in June of 2016 for each of the different fish

578 genotypes used in the experiment. DNA was then extracted from preserved fin clips from

579 experimental fish and microsatellite analysis was used to assign fish in the common garden design

580 to the genotypes they originated from.

581 Using recorded morphometrics, we determined the fish's condition (condition factor, CF),

582 hepatosomatic index (HIS), and immunological activation (splenosomatic index, SSI; head kidney

583 index, HKI) $[105,106]$. The parasite index (PI, the relative weight of the parasite in the host) was

584 also calculated if the fish was infected [107]. 
587

\section{Quantitative real-time PCR (RT-qPCR) of immune response genes}

Head kidney RNA was extracted with a NucleoSpin® 96 kit (Macherey-Nagel) following the manufacturer's protocol. RNA concentration and purity were determined spectrophotometrically (NanoDrop1000; Thermo Scientific). We used the Omniscript RT kit (Qiagen) according to the manual but used $0.2 \mu \mathrm{l}$ of a 4-unit RNase inhibitor (Qiagen) per reaction. Differences in gene transcription levels were tested using 96.96 Dynamic Array IFCs on a Biomark $^{\mathrm{TM}}$ HD system (Fluidigm) with EvaGreen as DNA intercalating dye on all the threespine sticklebacks used for this study as previously described. Briefly, we pre-amplified the cDNA samples by using TaqMan PreAmp Master Mix (Applied Biosystems) according to the manufacturer's protocol (14 cycles). All targets for a given sample were included in the same run and measured in technical triplicates. Inter-run calibrators and negative controls were included on each IFC. Melting curves were analysed with the Fluidigm Analysis software v.4.5.1.

\section{Bacterial microbiome composition and bacterial load}

DNA was extracted from intestines (In) and stomach (St) collected from 6 non-infected and 6 infected field caught individuals from Cheney lake. In addition, DNA was extracted from the intestine of 148 individuals from Walby (56 individuals), Wolf (38 individuals), and GPS (54 individuals) that were either (i) sham-exposed (42 individuals), (ii) exposed but non-infected by $S$. solidus from SKO (10 individuals), Walby (13 individuals) and Wolf (13 individuals), or (iii) exposed and successfully infected by $S$. solidus from SKO (18 individuals), Walby (28 individuals) and Wolf (24 individuals) (Table S1). The DNA of S. solidus worms from SKO (18 individuals), Walby (28 individuals) and Wolf (24 individuals) collected from ESI sticklebacks from Walby (27 individuals), Wolf (19individuals), and GPS (24 individuals) was also extracted. No Wolf Lake 
607 fish were successfully infected by SKO parasites, so that neither fish intestines nor S. solidus 608 worms were collected for this host-parasite combination.

DNA extraction was performed in the same manner for both field sampled and cross

610 infection samples using a Powerlyzer Powersoil Kit (Cat\#12855) with the following modifications

611 to the manufacturer's protocol. First, all tissue samples were placed in tough tubes (Qiagen CAT\#

612 19301) with 3mm glass beads (Sigma CAT\# S72683). To each tube, $750 \mu \mathrm{L}$ of bead beating

613 solution supplied with the extraction kit was added. Samples underwent three rounds of $20 \mathrm{sec}$

614 bead beating cycles and were kept on ice between each round. The homogenate was transferred to 615 the Qiagen supplied $0.1 \mathrm{~mm}$ bead tube and incubated with $60 \mu \mathrm{L}$ of $\mathrm{C} 1$ solution for $10 \mathrm{~min}$ at $60^{\circ} \mathrm{C}$. 616 After vortexing, centrifugation, and filtration with solution $\mathrm{C} 5,75 \mu \mathrm{L}$ of solution $\mathrm{C} 6$ was added to 617 the spin filter in a clean $2 \mathrm{~mL}$ tube and incubated at room temperature for $5 \mathrm{~min}$. Two rounds of 618 centrifugation at $10,000 \mathrm{~g}$ for $30 \mathrm{sec}$ were used to recover $150 \mu \mathrm{L}$ final DNA extract. Six negative 619 controls of molecular biology grade water were processed together with samples to identify kit 620 contaminants.

\section{$621 \quad$ Illumina Sequencing}

Sequencing was conducted by the Mr DNA lab in Shallowater, TX. Briefly, the 16S rDNA

623 V4-V5 hypervariable region was amplified with E. coli 515f and 806r primers (with barcode on 624 the forward primer) as specified by the Earth Microbiome [108-110]. After amplification, PCR 625 products were checked in $2 \%$ agarose gel to determine the success of amplification and the relative 626 intensity of bands. PCR products were pooled together in equal proportions based on their 627 molecular weight and DNA concentrations. Pooled samples were purified before Illumina DNA 628 library preparation. Illumina sequencing was employed to obtain $250 \mathrm{bp}$ paired end reads on a 
MiSeq following the manufacturer's guidelines. Sequence data were initially processed to join forward and reverse reads and remove barcodes.

Sequencing of field collected samples yielded an average of 43,645 ( \pm SE 16,027) and $10,800( \pm$ SE 8,824$)$ high quality paired end reads for G. aculeatus and $S$. solidus tissue samples respectively. Sequencing depth of control samples was higher and yielded an average of 83,875 $( \pm$ SE 10,080$)$ high quality reads. Sequencing of cross infection samples yielded an average of $53,827( \pm$ SE 24,004) high quality paired end reads for fish samples, 55,308 $( \pm$ SE 28,426$)$ for $S$. solidus samples, and 69,273 ( \pm SE 16,277) high quality reads for kit control samples. Due to the large number of samples sequenced, multiple sequencing runs were processed. In order to extract the same Amplicon Sequence Variants (ASVs) for all sequencing runs, each run was demultiplexed, sequences quality scores were controlled, and sequences were trimmed at the same quality threshold to obtain sequences of a uniform 200 bp length. ASVs were resolved to avoid applying the arbitrary 97\% threshold that defines molecular OTUs but has limited biological meaning [111]. Taxonomy was assigned to each of the ASVs using a pretrained Naïve-Bayes Classifier trained on the GreenGenes 13_8 99\% OTUs.

Swabs of the body cavity of G. aculeatus and surface bacteria from S. solidus used as controls had a significantly different microbiome from fish and parasite samples (Figure S4). Similarly, blank controls used for the cross-infection experiments had a different microbiome from fish and parasite samples. ASVs abundant in all control samples were extremely similar to common bacterial contaminants of the MoBio kit used for bacterial DNA extraction and were removed from the analysis [112-114]. ASVs classified only to the domain level or listed as unidentified were blasted against the GenBank reference database and sequences that aligned to non-bacterial organisms were removed. We further decontaminated samples using the Decontam 
652 package in $\mathrm{R}$ which has been found to be successful at decontaminating data sets with low biomass

653 samples $[115,116]$.

654 qPCR of 16S gene

655 Real-time qPCR was conducted using the QuantStudio 6 Flex RT PCR System (Fisher 656 Scientific). The qPCR mixture $(20 \mu \mathrm{L})$ was composed of $10 \mu \mathrm{L}$ of SYBR green master mix 657 (Thermofisher CAT\# 4309155), 4.8 $\mu \mathrm{L}$ molecular biology grade water (Thermofisher 658 CAT\#46000), 0.6 $\mu \mathrm{L}$ forward primer, $0.6 \mu \mathrm{L}$ reverse primer, and $4 \mu \mathrm{L}$ DNA. Two $16 \mathrm{~S}$ primers were 659 used (Forward: TCCTACGGGAGGCAGCAGT, Reverse:

660 GACTACCAGGGTATCTAATCCTGTT [108] and Forward: GTGSTGCAYGGYTGTCGTCA, 661 Reverse: ACGTCRTCCMCACCTTCCTC [109]) and compared to an E. coli standard for which 662 cell count was obtained using a hemocytometer. After quantitative PCR, CT values were averaged 663 and used to calculate the relative number of cells using the slope formula generated with the $E$. 664 coli standard curve. Even though the number of $16 \mathrm{~S}$ gene copies per bacterial cell cannot be 665 controlled, the qPCR assay provides a good proxy for bacterial abundance [117-118].

666

667 Diversity metrics and statistical analyses

668 Diversity calculations and field sample analysis

669 All data analyses were performed using QIIME2 and the Phyloseq, and lmertest packages 670 in R [119-122]. First, Shannon, Simpson, and Fisher alpha diversity metrics were used to estimate 671 within sample diversity and compared using Tukey comparison of means. Beta diversity was 672 calculated with Weighted and Unweighted Unifrac Distances. Principal coordinate analysis 673 (PCoA) on Unifrac distances was performed to look for clustering of samples in unconstrained 
674 multivariate space. Coordinates along PCoA, PERMANOVA, and PERMDISP were used to 675 compare differences between sample groups. Heatmaps were generated using Excel.

\section{Cross infection microbiome models}

In order to account for all potential factors, statistical analyses for the cross infection 679 experiment were conducted using linear mixed models using the lmertest package in $\mathrm{R}$ using each 680 of the different microbiome indices (Shannon, Simpson, Fisher, PCoA1, PCoA2, PCoA3, and

681 bacterial load) as the dependent variable for a given model [120]. We began by testing the impact 682 on fish population in control non-exposed fish (CNI) (Figure 2A). We conducted model selection starting with the most complex model which included fish population, sex and their interactions

684 as fixed factors and tank and round as random factors down to the simplest model which included 685 only fish population as a fixed factor and tank and round as random factors. We then calculated 686 the AIC for each of the models and chose the model with the lowest AIC to run. If the AIC values 687 differed by less than a value of two for the best fit models, we then conducted ANOVA to see if 688 there was significant variance between the best fit model. If there were significant differences, the 689 model with the lowest AIC was selected. If there were no significant differences the simplest model was selected. P-values were derived from the selected model using least squared means (LSM) 691 calculated with the lsmeans package [122].

To investigate the impact of infection status on these indices, we used infection status,

693 which included the levels CNI (control non-infected), ENI (exposed non-infected), and ESI 694 (exposed and successfully infected), sex, and their interaction as potential fixed factors, and 695 random factors were fish population, parasite population, tank, and round. Model selection was 696 performed as described above and the best-fit model was chosen to calculate p-values (Figure 2). 
697 Following this we tested the impact of fish and parasite population in exposed non-infected fish 698 (ENI), in successfully infected fish (ESI), and parasites (Ss). After conducting model selection as 699 described above, we tested for the role of fish population and parasite population separately (Figure 700 2). When testing fish population effects, we used fish population, sex, and their interaction as fixed 701 factors, and random factors were parasites population, tank, and round. When testing parasite 702 population effects, we used parasite population, sex, and their interaction as fixed factors and 703 random factors were fish population, tank, and round. Model selection was conducted each time 704 and the best fit model was chosen to calculate p-values.

705

Differentially abundant bacteria

707 We used the DESeq2 package in R [123] to identify differentially abundant bacteria.

708 From field samples, DESeq2 was used to compare bacterial abundance between threespine

709 sticklebacks and S. solidus, between intestine and stomach, and between non-infected and 710 infected fish. From the cross-infection experiment, DESeq2 was used to test variations in

711 bacterial abundance between threespine sticklebacks and S. solidus, depending on infection

712 status (CNI, ENI and ESI), fish population (for Ss, CNI, ENI and ESI), parasite population (for

713 Ss, ENI and ESI) while controlling for tank, round effects, and fish and parasite population as

714 appropriate by including these variables in the models.

715

\section{Correlation between gene expression and microbiome parameters}

We assessed whether microbiome diversity metrics and the relative abundance of most abundant bacterial families correlated with phenotypic data and gene expression data. First, we

719 conducted a principal component analysis (PCA) with on 25 genes including three regulatory 
genes (abtb1, kat2a, mapk13) and 22 immune related genes from innate immunity (marco, $\mathrm{mst} 1 \mathrm{ra}$, mif, tnfrl, saall, tlr2, csf3r, p22 $2^{\text {phox }}$, nkef-b, slal, cd97), adaptive immunity (stat4, stat6, igm, cd83, foxp3, il-16, mhcII, tcr- $\beta$ ) and the complement system $(c f b, c 7, c 9)$ for all samples for which the microbiome composition had been assessed. Next, we conducted a correlation analysis including all microbiome diversity metrics (Log Cells, Fisher, Shannon, Simpson, weighted unifrac PC1 to 3 and unweighted unifrac PC 1 to 3 ) and the 30 most abundant bacterial families, together with the three top gene PCA coordinates and relative expression of all 25 tested genes. We used Spearman's rank correlation because it performs better with log transformed data and microbiome relative abundance and allows comparisons between datasets with different distribution profiles. Due to multiple testing, $\mathrm{p}$ values were corrected for multiple comparisons with a Bonferroni correction.

\section{$7 \quad$ Acknowledgements}

Not applicable.

\section{References}

1. De Palma, G., et al., Microbiota and host determinants of behavioural phenotype in maternally separated mice. Nature communications, 2015. 6(1): p. 1-13.

2. Stencel, A. and D.M. Wloch-Salamon, Some theoretical insights into the hologenome theory of evolution and the role of microbes in speciation. Theory in Biosience, 2018. 137(2): p. 197-206.

3. Li, M., et al., Symbiotic gut microbes modulate human metabolic phenotypes. Proceedings of the National Academy of Sciences, 2008. 105(6): p. 2117-2122.

4. $\quad$ Busby, P.E., et al., Research priorities for harnessing plant microbiomes in sustainable agriculture. PLoS biology, 2017. 15(3): p. e2001793.

5. Mueller, U.G. and J.L. Sachs, Engineering microbiomes to improve plant and animal health. Trends in microbiology, 2015. 23(10): p. 606-617.

6. Greer, R., et al., Investigating a holobiont: Microbiota perturbations and transkingdom networks. 2016. 7(2): p. 126-135.

7. $\quad$ Ursell, L.K., et al., Defining the human microbiome. 2012. 70(suppl_1): p. S38-S44.

8. Turnbaugh, P.J., et al., The human microbiome project. 2007. 449(7164): p. 804-810.

9. Maslowski, K.M. and C.R. Mackay, Diet, gut microbiota and immune responses. Nature immunology, 2011. 12(1): p. 5-9.

10. Chilton, S.N., et al., The effects of diet and the microbiome on reproduction and longevity: a comparative review across 5 continents. 2015. 5(3). 
11. Dietert, R.R., The microbiome-immune-host defense barrier complex (microimmunosome) and developmental programming of noncommunicable diseases. Reproductive Toxicology, 2016.

12. Li, R.W., et al., Alterations in the porcine colon microbiota induced by the gastrointestinal nematode Trichuris suis. Infect Immun, 2012. 80(6): p. 2150-2157.

13. Holm, J.B., et al., Chronic Trichuris muris infection decreases diversity of the intestinal microbiota and concomitantly increases the abundance of lactobacilli. PLoS One, 2015. 10(5): p. e0125495.

14. Itthitaetrakool, U., et al., Chronic Opisthorchis viverrini Infection Changes the Liver Microbiome and Promotes Helicobacter Growth. PloS ONE, 2016. 11(11): p. e0165798.

15. Theis, K., et al., Getting the hologenome concept right: an eco-evolutionary framework for hosts and their microbiomes. Msystems, 2016. 1(2).

16. Dheilly, N., Holobiont-Holobiont interactions: redefining host-parasite interactions. PLOS Pathogens, 2014. 10(7).

17. McKenney, E.A., et al., Alteration of the rat cecal microbiome during colonization with the helminth Hymenolepis diminuta. Gut Micro, 2015. 6(3): p. 182-193.

18. Li, R.W., et al., The effect of helminth infection on the microbial composition and structure of the caprine abomasal microbiome. Sci Rep, 2016. 6.

19. Plieskatt, J.L., et al., Infection with the carcinogenic liver fluke Opisthorchis viverrini modifies intestinal and biliary microbiome. FASEB J, 2013. 27(11): p. 4572-4584.

20. Zaiss, M. and N.J.P.i. Harris, Interactions between the intestinal microbiome and helminth parasites. 2016. 38(1): p. 5-11.

21. Kostic, A.D., R.J. Xavier, and D. Gevers, The microbiome in inflammatory bowel disease: current status and the future ahead. Gastroenterology, 2014. 146(6): p. 1489-1499.

22. Taylor, M.J., H.F. Cross, and K. Bilo, Inflammatory responses induced by the filarial nematode Brugia malayi are mediated by lipopolysaccharide-like activity from endosymbiotic Wolbachia bacteria. J Exp Med, 2000. 191(8): p. 1429-1436.

23. Cooke, A.J.C.o.i.r., Parasitic worms and inflammatory disease. 2012. 24(4): p. 394-400.

24. Broadhurst, M.J., et al., Therapeutic helminth infection of macaques with idiopathic chronic diarrhea alters the inflammatory signature and mucosal microbiota of the colon. 2012. 8(11).

25. Rosa, B.A., et al., Differential human gut microbiome assemblages during soil-transmitted helminth infections in Indonesia and Liberia. 2018. 6(1): p. 33.

26. Lee, S.C., et al., Helminth colonization is associated with increased diversity of the gut microbiota. PLoS Negl Trop Dis, 2014. 8(5): p. e2880.

27. Walk, S.T., et al., Alteration of the murine gut microbiota during infection with the parasitic helminth Heligmosomoides polygyrus. J Inflamm Bowel Dis Disord, 2010. 16(11): p. 1841-1849.

28. Houlden, A., et al., Chronic Trichuris muris infection in C57BL/6 mice causes significant changes in host microbiota and metabolome: effects reversed by pathogen clearance. 2015. 10(5).

29. Cooper, P.J. and C.A. Figuieredo, Immunology of Ascaris and immunomodulation, in Ascaris: the neglected parasite. 2013, Elsevier. p. 3-19.

30. Cantacessi, C., et al., Impact of experimental hookworm infection on the human gut microbiota. J Infect Dis, 2014. 210(9): p. 1431-1434. 
31. Leung, J.M., A.L. Graham, and S.C. Knowles, Parasite-microbiota interactions with the vertebrate gut: synthesis through an ecological lens. Frontiers in microbiology, 2018. 9: p. 843.

32. Kurtz, J., et al., Major histocompatibility complex diversity influences parasite resistance and innate immunity in sticklebacks. Philos Trans R Soc Lond B Biol Sci, 2004. 271(1535): p. 197-204.

33. Piecyk, A., O. Roth, and M. Kalbe, Specificity of resistance and geographic patterns of virulence in a vertebrate host-parasite system. BMC evolutionary biology, 2019. 19(1): p. 80.

34. Mackinnon, M.J. and A.F.J.P.b. Read, Immunity promotes virulence evolution in a malaria model. 2004. 2(9).

35. Alizon, S. and M.J.T.A.N. van Baalen, Multiple infections, immune dynamics, and the evolution of virulence. 2008. 172(4): p. E150-E168.

36. Ling, F., et al., The gut microbiota response to helminth infection depends on host sex and genotype. The ISME Journal, 2020. 14(5): p. 1141-1153.

37. Knutie, S.A., et al., Early-life disruption of amphibian microbiota decreases later-life resistance to parasites. Nat Commun, 2017. 8(1): p. 86.

38. Aivelo, T. and A. Norberg, Parasite-microbiota interactions potentially affect intestinal communities in wild mammals. Journal of Animal Ecology, 2018. 87(2): p. 438-447.

39. Brosschot, T. and L. Reynolds, The impact of a helminth-modified microbiome on host immunity. Mucosal immunology, 2018. 11(4): p. 1039-1046.

40. Cayrol, C. and J.-P.J.C.o.i.i. Girard, IL-33: an alarmin cytokine with crucial roles in innate immunity, inflammation and allergy. 2014. 31: p. 31-37.

41. Ziegler, S.F. and D.J.N.i. Artis, Sensing the outside world: TSLP regulates barrier immunity. 2010. 11(4): p. 289-293.

42. Balic, A., et al., Selective maturation of dendritic cells by Nippostrongylus brasiliensissecreted proteins drives Th2 immune responses. 2004. 34(11): p. 3047-3059.

43. Cervi, L., et al., Cutting edge: dendritic cells copulsed with microbial and helminth antigens undergo modified maturation, segregate the antigens to distinct intracellular compartments, and concurrently induce microbe-specific Th1 and helminth-specific Th2 responses. 2004. 172(4): p. 2016-2020.

44. Segura, M., et al., Impairment of dendritic cell function by excretory-secretory products: a potential mechanism for nematode-induced immunosuppression. 2007. 37(7): p. 18871904.

45. Massacand, J.C., et al., Helminth products bypass the need for TSLP in Th2 immune responses by directly modulating dendritic cell function. 2009. 106(33): p. 13968-13973.

46. Wesemann, D.R., Microbes and B cell development, in Advances in immunology. 2015, Elsevier. p. 155-178.

47. Kaplan, J.L., H.N. Shi, and W.A.J.P.r. Walker, The role of microbes in developmental immunologic programming. 2011. 69(6): p. 465-472.

48. Bauché, D., J.C.J.C. Marie, and t. immunology, Transforming growth factor $\beta$ : a master regulator of the gut microbiota and immune cell interactions. 2017. 6(4): p. e136.

49. Pandiyan, P., et al., Microbiome dependent regulation of Tregs and Th17 cells in mucosa. 2019. 10: p. 426.

50. Ichinohe, T., et al., Microbiota regulates immune defense against respiratory tract influenza A virus infection. 2011. 108(13): p. 5354-5359. 
51. Taylor, M.J., C. Bandi, and A. Hoerauf, Wolbachia. Bacterial endosymbionts of filarial nematodes. Adv Parasitol, 2005. 60: p. 245-284.

52. Lawrence, S.A. and R. Poulin, Detection of the bacterial endosymbiont Neorickettsia in a New Zealand digenean. J Parasitol Res, 2016. 115(11): p. 4275-4279.

53. Pusterla, N., et al., Digenetic trematodes, Acanthatrium sp. and Lecithodendrium sp., as vectors of Neorickettsia risticii, the agent of Potomac horse fever. J Helminthol, 2003. 77(04): p. 335-339.

54. Vaughan, J.A., V.V. Tkach, and S.E. Greiman, Neorickettsial endosymbionts of the digenea: diversity, transmission and distribution, in Adv Parasitol. 2012, Elsevier. p. 253297.

55. Fischer, K., et al., Ultrastructure and localization of Neorickettsia in adult digenean trematodes provides novel insights into helminth-endobacteria interaction. Parasit Vectors, 2017. 10(1): p. 177.

56. Hahn, M.A. and N.M. Dheilly, Experimental Models to Study the Role of Microbes in HostParasite Interactions. Frontiers in Microbiology, 2016. 7(1300).

57. Dheilly, N.M., et al., Parasite microbiome project: Grand challenges. 2019. 15(10).

58. Sinnathamby, G., et al., The bacterial community associated with the sheep gastrointestinal nematode parasite Haemonchus contortus. PLOS One, 2018. 13(2).

59. White, E., et al., Manipulation of host and parasite microbiotas: Survival strategies during chronic nematode infection. Science advances, 2018. 4(3): p. eaap7399.

60. Chng, K.R., et al., Tissue microbiome profiling identifies an enrichment of specific enteric bacteria In Opisthorchis viverrini associated cholangiocarcinoma. EBioMedicine, 2016.

61. Poulin, R., F. Jorge, and N.M.J.F.i.M. Dheilly, Persistence of a core microbiome through the ontogeny of a multi-host parasite. 2020. 11: p. 954.

62. Hammerschmidt, K. and J. Kurtz, Exp Parasitol: Establishment of tapeworms in sticklebacks - fast food or fast lane? Exp Parasitol, 2007. 116(2): p. 142-149.

63. Jorge, F., N. Dheilly, and R. Poulin, Persistence of a Core Microbiome Through the Ontogeny of a Multi-Host Parasite. Frontiers in Microbiology, 2020. 11: p. 954.

64. Chng, K., et al., Tissue microbiome profiling identifies an enrichment of specific enteric bacteria in Opisthorchis viverrini associated cholangiocarcinoma. EBioMedicine, 2016. 8: p. 195-202.

65. Brooks, A., et al., Phylosymbiosis: relationships and functional effects of microbial communities across host evolutionary history. PLoS biology, 2016. 14(11): p. e2000225.

66. Sprehn, G., et al., Landscape genetics of Schistocephalus solidus parasites in threespine stickleback (Gasterosteus aculeatus) from Alaska. PLOS One, 2015. 10(4).

67. Marques, D., et al., Genomics of rapid incipient speciation in sympatric threespine stickleback. PLOS Genetics, 2016. 12(2): p. e1005887.

68. Goldstein, E., et al., Desulfovibrio desulfuricans bacteremia and review of human Desulfovibrio infections. Journal of clinical microbiology, 2003. 41(6): p. 2752-2754.

69. Kuever, J., F. Rainey, and F. Widdel, Desulfovibrio. Bergey's Manual of Systematics of Archaea Bacteria, 2015: p. 1-17.

70. Rodrick, G., Indigenous pathogens: Vibrionaceae, in Microbiology of Marine Food Products. 1991, Springer. p. 285-300. 
71. Itthitaetrakool, U., et al., Chronic Opisthorchis viverrini Infection Changes the Liver Microbiome and Promotes Helicobacter Growth. PLOS One, 2016. 11(11): p. e0165798e0165798.

72. Everts, B., et al., Helminths and dendritic cells: sensing and regulating via pattern recognition receptors, Th2 and Treg responses. European journal of immunology, 2010. 40(6): p. 1525-1537.

73. Piecyk, A., M. Ritter, and M. Kalbe, The right response at the right time: Exploring helminth immune modulation in sticklebacks by experimental coinfection. Molecular Ecology, 2019. 28(10): p. 2668-2680.

74. Kurtz, J., et al., Major histocompatibility complex diversity influences parasite resistance and innate immunity in sticklebacks. Proceedings of the Royal Society of London. Series B: Biological Sciences, 2004. 271(1535): p. 197-204.

75. Lindenberg, F., et al., Expression of immune regulatory genes correlate with the abundance of specific Clostridiales and Verrucomicrobia species in the equine ileum and cecum. Scientific reports, 2019. 9(1): p. 1-10.

76. Ellekilde, M., et al., Characterization of the gut microbiota in leptin deficient obese miceCorrelation to inflammatory and diabetic parameters. Research in veterinary science, 2014. 96(2): p. 241-250.

77. Lundberg, R., et al., Gastrointestinal microbiota and local inflammation during oxazoloneinduced dermatitis in BALB/cA mice. Comparative Medicine, 2012. 62(5): p. 371-380.

78. Bendtsen, K.M.B., et al., Gut microbiota composition is correlated to grid floor induced stress and behavior in the BALB/c mouse. PLOS One, 2012. 7(10): p. e46231.

79. Golubeva, A.V., et al., Prenatal stress-induced alterations in major physiological systems correlate with gut microbiota composition in adulthood. Psychoneuroendocrinology, 2015. 60: p. 58-74.

80. Sundin, J., et al., Gut Microbiota Correlate With Psychological Distress and Intestinal Lymphocyte Composition in Post-Infectious Irritable Bowel Syndrome Patients. Gastroenterology, 2015. 148(4): p. S584-S584.

81. Claesson, M.J., et al., Gut microbiota composition correlates with diet and health in the elderly. Nature, 2012. 488(7410): p. 178-184.

82. Abusleme, L., et al., The subgingival microbiome in health and periodontitis and its relationship with community biomass and inflammation. The ISME Journal, 2013. 7(5): p. 1016-1025.

83. Baldani, J.I., et al., The Family Rhodospirillaceae, in The Prokaryotes: Alphaproteobacteria and Betaproteobacteria, E. Rosenberg, et al., Editors. 2014, Springer Berlin Heidelberg: Berlin, Heidelberg. p. 533-618.

84. Layland, L.E. and I. Ludwig-Portugall, TLRs, Treg, and B cells, an interplay of regulation during helminth infection. Frontiers in immunology, 2012. 3: p. 8.

85. King, K. and M. Bonsall, The evolutionary and coevolutionary consequences of defensive microbes for host-parasite interactions. BMC Evolutionay Biology, 2017. 17(1): p. 190202.

86. Ling, F., et al., The gut microbiota response to helminth infection depends on host sex and genotype. 2020: p. 1-13.

87. Smith, C., et al., Dietary input of microbes and host genetic variation shape amongpopulation differences in stickleback gut microbiota. The ISME Journal, 2015. 9(11): p. 2515-2526. 
88. Steury, R., et al., Population genetic divergence and environment influence the gut microbiome in Oregon threespine stickleback. Genes, 2019. 10(7): p. 484.

89. Small, C., et al., Host genotype and microbiota contribute asymmetrically to transcriptional variation in the threespine stickleback gut. Genome biology and evolution, 2017. 9(3): p. 504-520.

90. Kalbe, M., et al., Reciprocal cross infection of sticklebacks with the diphyllobothriidean cestode Schistocephalus solidus reveals consistent population differences in parasite growth and host resistance. Parasite Vectors, 2016. 9(1): p. 130-142.

91. Poulin, R., Parasite manipulation of host personality and behavioural syndromes. Journal of Experimental Biology, 2013. 216(1): p. 18-26.

92. Weber, J.N., et al., Resist globally, infect locally: A transcontinental test of adaptation by stickleback and their tapeworm parasite. The American Naturalist, 2017. 189(1): p. 43-57.

93. Rausch, S., et al., Parasitic nematodes exert antimicrobial activity and benefit from microbiota-driven support for host immune regulation. Frontiers in immunology, 2018. 9: p. 2282.

94. Haase, D., et al., Specific gene expression responses to parasite genotypes reveal redundancy of innate immunity in vertebrates. PLOS One, 2014. 9(9): p. e108001.

95. Moloo, A. Neglected Tropical Diseases. 2018; Available from: http://www.who.int/neglected_diseases/diseases/en/.

96. Zaiss, M.M., et al., The intestinal microbiota contributes to the ability of helminths to modulate allergic inflammation. Immunity, 2015. 43(5): p. 998-1010.

97. Brosschot, T. and L. Reynolds, The impact of a helminth-modified microbiome on host immunity. Mucosal immunology, 2018. 11(4): p. 1039-1046.

98. Hahn, M., et al., Characterization of viruses in a tapeworm: phylogenetic position, vertical transmission, and transmission to the parasitized host. ISME, 2020: p. 1-13.

99. Dheilly, N., et al., Parasite microbiome project: systematic investigation of microbiome dynamics within and across parasite-host interactions. MSystems, 2017. 2(4): p. e0005017.

100. Griffiths, R., et al., DNA sex identification in the three-spined stickleback. Journal of Fish Biology, 2000. 57(5): p. 1331-1334.

101. Smyth, J., Studies on tapeworm physiology: I. The cultivation of Schistocephalus solidus in vitro. Journal of Experimental Biology, 1946. 23(1): p. 47-70.

102. Wedekind, C., The infectivity, growth, and virulence of the cestode Schistocephalus solidus in its first intermediate host, the copepod Macrocyclops albidus. Parasitology, 1997. 115(3): p. 317-324.

103. Imsland, A.K. and O.D.B. Jónsdóttir, Linking population genetics and growth properties of Atlantic cod. Reviews in Fish Biology and Fisheries, 2003. 13(1): p. 1-26.

104. Conover, D., et al., Spatial and temporal scales of adaptive divergence in marine fishes and the implications for conservation. Journal of fish biology, 2006. 69: p. 21-47.

105. Frischknecht, M., The breeding colouration of male three-spined sticklebacks (Gasterosteus aculeatus) as an indicator of energy investment in vigour. Evolutionary Ecology, 1993. 7(5): p. 439-450.

106. Chellappa, S., et al., Condition factor and hepatosomatic index as estimates of energy status in male three-spined stickleback. Journal of Fish Biology, 1995. 47(5): p. 775-787. 
107. Arme, C. and R.W. Owen, Infections of the three-spined stickleback, Gasterosteus aculeatus L., with the plerocercoid larvae of Schistocephalus solidus (Müller, 1776), with special reference to pathological effects. Parasitology, 1967. 57(02): p. 301-314.

108. Apprill, A., et al., Minor revision to V4 region SSU rRNA 806R gene primer greatly increases detection of SAR11 bacterioplankton. 2015. 75(2): p. 129-137.

109. Parada, A.E., D.M. Needham, and J.A.J.E.m. Fuhrman, Every base matters: assessing small subunit rRNA primers for marine microbiomes with mock communities, time series and global field samples. 2016. 18(5): p. 1403-1414.

110. Gilbert, J.A., J.K. Jansson, and R. Knight, The Earth Microbiome project: successes and aspirations. BMC biology, 2014. 12(1): p. 69.

111. Callahan, B.J., P.J. McMurdie, and S.P. Holmes, Exact sequence variants should replace operational taxonomic units in marker-gene data analysis. The ISME journal, 2017. 11(12): p. 2639-2643.

112. Glassing, A., et al., Inherent bacterial DNA contamination of extraction and sequencing reagents may affect interpretation of microbiota in low bacterial biomass samples. Gut pathogens, 2016. 8(1): p. 24.

113. Salter, S.J., et al., Reagent and laboratory contamination can critically impact sequencebased microbiome analyses. BMC biology, 2014. 12(1): p. 87.

114. Weiss, S., et al., Tracking down the sources of experimental contamination in microbiome studies. Genome biology, 2014. 15(12): p. 564.

115. Karstens, L., et al., Controlling for Contaminants in Low-Biomass $16 S$ rRNA Gene Sequencing Experiments. mSystems, 2019. 4(4): p. e00290-19.

116. Davis, N.M., et al., Simple statistical identification and removal of contaminant sequences in marker-gene and metagenomics data. Microbiome, 2018. 6(1): p. 226.

117. Boshier, F.A.T., et al., Complementing 16S rRNA Gene Amplicon Sequencing with Total Bacterial Load To Infer Absolute Species Concentrations in the Vaginal Microbiome. 2020. 5(2).

118. Horz, H., et al., Evaluation of universal probes and primer sets for assessing total bacterial load in clinical samples: general implications and practical use in endodontic antimicrobial therapy. Journal of clinical microbiology, 2005. 43(10): p. 5332-5337.

119. McMurdie, P.J. and S. Holmes, phyloseq: an $R$ package for reproducible interactive analysis and graphics of microbiome census data. PloS one, 2013. 8(4): p. e61217.

120. Kuznetsova, A., P.B. Brockhoff, and R.H.B.J.J.o.s.s. Christensen, lmerTest package: tests in linear mixed effects models. 2017. 82(13): p. 1-26.

121. Bolyen, E., et al., QIIME 2: Reproducible, interactive, scalable, and extensible microbiome data science. 2018, PeerJ Preprints.

122. Lenth, R.V.J.J.S.S., Using lsmeans. 2017. 69: p. 1-33.

123. Anders, S. and W. Huber, Differential expression of RNA-Seq data at the gene level-the DESeq package. Heidelberg, Germany: European Molecular Biology Laboratory (EMBL), 2012.

\section{Figure Legends}

Figure 1: Summary of experimental design. (A) The map displays all sampling locations. The field study was conducted on sticklebacks and S. solidus from one location in Alaska (Cheney). 
1022 The three genotypes of stickleback hosts (Walby (W), Wolf (w) and GPS(G)) and three genotypes 1023 of S. solidus (Walby (W), Wolf (w) and SKO (S)) used for experimental infection were collected 1024 from lakes in Alaska and Europe. (B) Design matrix showing the organization of rounds and tanks 1025 based on fish genotype (denoted by letters) and parasite genotype (denoted by numbers). A total 1026 of 36 tanks were used. One example is squared in red. (C) Each of the tanks contained three sham 1027 control fish (left) from Wolf (Red Tail), Walby (Green Tail), and GPS (Blue Tail) separated by a 1028 mesh divider from 5 Wolf fish , 5 GPS fish, and 4 Walby fish that had been exposed to parasites 1029 (right). (D) Upon dissection, the success of infection was assessed, and exposed individuals were 1030 classified as exposed non-infected (ENI) or exposed successfully infected (ESI). All successfully 1031 infected fish were processed, and corresponding ENI and control non-infected fish from the same 1032 tanks and fish origin were processed as controls.

1034 Figure 2: Stickleback bacterial community composition vary with exposure, infection status, 1035 host genotype and parasite genotype as revealed by LMMs. (A) Among the control non1036 infected fish, the microbiome composition varies with fish genotype. Differences were found 1037 between Walby $(\mathrm{n}=17)$ and GPS fish $(\mathrm{n}=15)$ (Weighted Unifrac, Axes 1 LMM $\mathrm{p}=0.038$; and Axis 1038 3, Unweighted Unifrac, Axis 3, p=0.028) and between Wolf $(\mathrm{n}=10)$ and GPS fish (Unweighted 1039 Unifrac Axis 1, p=0.024) . (B) Exposure and infection impact the microbial diversity and 1040 composition. Exposure impacts beta diversity (Weighted Unifrac, Axis 3, p=0.031), whereas 1041 successful infection is associated with changes in bacterial load $(\mathrm{p}=0.01)$ and alpha diversity 1042 (Shannon $\mathrm{p}=0.039$, Simpson $\mathrm{p}=0.013$ ). (C) Among exposed but non-infected fish, the microbiome 1043 diversity and composition vary with host genotype and parasite genotype. Differences were found 1044 between Wolf $(n=9)$ and GPS $(n=14)$ fish (Fisher $\mathrm{p}=0.008$, Unweighted Unifrac Axis $1 \mathrm{p}=0.005$, 
and $3 \mathrm{p}=0.013$ ), between Walby $(\mathrm{n}=10)$ and Wolf fish (Shannon $\mathrm{p}=0.034$, Simpson $\mathrm{p}=0.020$, Fisher $\mathrm{p}=0.004$, Unweighted Unifrac Axis $1 \mathrm{p}=0.006$ ) and between Walby and GPS fish (Unweighted Unifrac Axis $3 \mathrm{p}=0.041$ ). Differences were found between alpha diversity of fish exposed to Walby and SKO parasites (Fisher p=0.018). (D) Among infected sticklebacks, microbiome composition varies with parasite origin and host-parasite co-evolutionary history. Fish infected with SKO $(n=18)$ parasites had higher bacterial load than fish infected with Walby $(n=27, p=0.010)$ and Wolf parasites $(n=25, p=0.001)$. Unweighted Unifrac distance revealed distinct clustering of fish infected with SKO parasites and fish infected with Walby or Wolf parasites (Axis 2, p=0.046 and $\mathrm{p}=0.047$ respectively). (E) Within parasites, microbiome composition varies with parasite origin. Weighted Unifrac distance revealed distinct clustering of the microbiome of SKO $(n=18)$ and Walby ( $\mathrm{n}=27$ ) parasites (Axis $3, \mathrm{p}=0.035$ ). The bottom and top edges of the boxes mark the $25^{\text {th }}$ and $75^{\text {th }}$ percentiles. The horizontal line denotes the median. Whiskers mark the range of the data excluding outliers (dots).

Figure 3: Bacterial taxa at multiple taxonomic levels vary in relative abundance and prevalence between threespine stickleback gut and $S$. solidus plerocercoids. The heatmaps depict the prevalence of the bacterial families (A) or ASVs (B) at different detection thresholds (defined in terms of minimum relative abundance) for successfully infected (ESI) sticklebacks, and corresponding S. solidus. A/ 30 most abundant bacterial families across all samples. B/ 30 most abundant ASVs in S. solidus. The phylum is provided as a color code next to the taxa name.

\section{Figure 4: The host phenotype depends on the composition of the microbiome of the host and}

of its parasite. A. Correlation heatmap depicting gene expression - microbial profile correlations. 
1068 Color of the squares indicate the strength of Spearman's rank correlation. Asterisks indicate 1069 significance of the correlation $(* \mathrm{q}<0.05 ; * * \mathrm{q}<0.05 ; * * * \mathrm{q}<0.001)$. B. Comparison of correlations 1070 between gene PC1 and uwuPC3 depending on infection status, host genotype and parasite 1071 genotype. Significant differences are observed when the confidence interval at the lower and/or 1072 higher values do not overlap with the mean of the other one. C. A. Correlation heatmap between 1073 the expression of genes, and phenotypic indices (HI, Hepatosomatic index; SI, Splenosomatic 1074 index, HKI Head-kidney index; BC, Body condition) and the relative abundance in the most 1075 abundant acterial families in the host G. aculeatus, and its parasite S. solidus. Color indicate the 1076 strength of Spearman's rank correlation. The complete figure with indication of significance is 1077 available in Figure S12. Significant Spearman's rank correlation (with FDR <0.05) are plotted in 1078 (D) between the relative abundance in Streptococcaceae, an unknown Actynomicetales and 1079 Aerococcaceae in the host with Heptaosomatic index $(\mathrm{N}=145)$, in (E) between the relative 1080 abundance of Synechoccophycidae in fish and the relative expression of foxp3, ill6, cd97 and stat6 $1081(\mathrm{~N}=145)$, in $(\mathrm{F})$ between the relative abundance in Lactobacillaceae in fish and the relative 1082 expression of foxp3, cd97 and stat6 $(\mathrm{N}=145)$, in $(G)$ between the relative abundance in 1083 Pseudanabaenaceae in fish and the relative expression of foxp3, cd97 and stat4 (N=145), in $(\mathrm{H})$ 1084 between the relative abundance of Vibrionaceae in fish and relative expression of of foxp3, ill6, 1085 cd97 and stat6 $(\mathrm{N}=145)$ and in (I) between the relative abundance of Chloroflexi in the parasite 1086 and relative abundance in foxp3, thfr1, cd97, stat6, and marco $(\mathrm{N}=69)$. The significant Spearman 1087 correlation, after Bonferroni correction, are provided on each plot. 


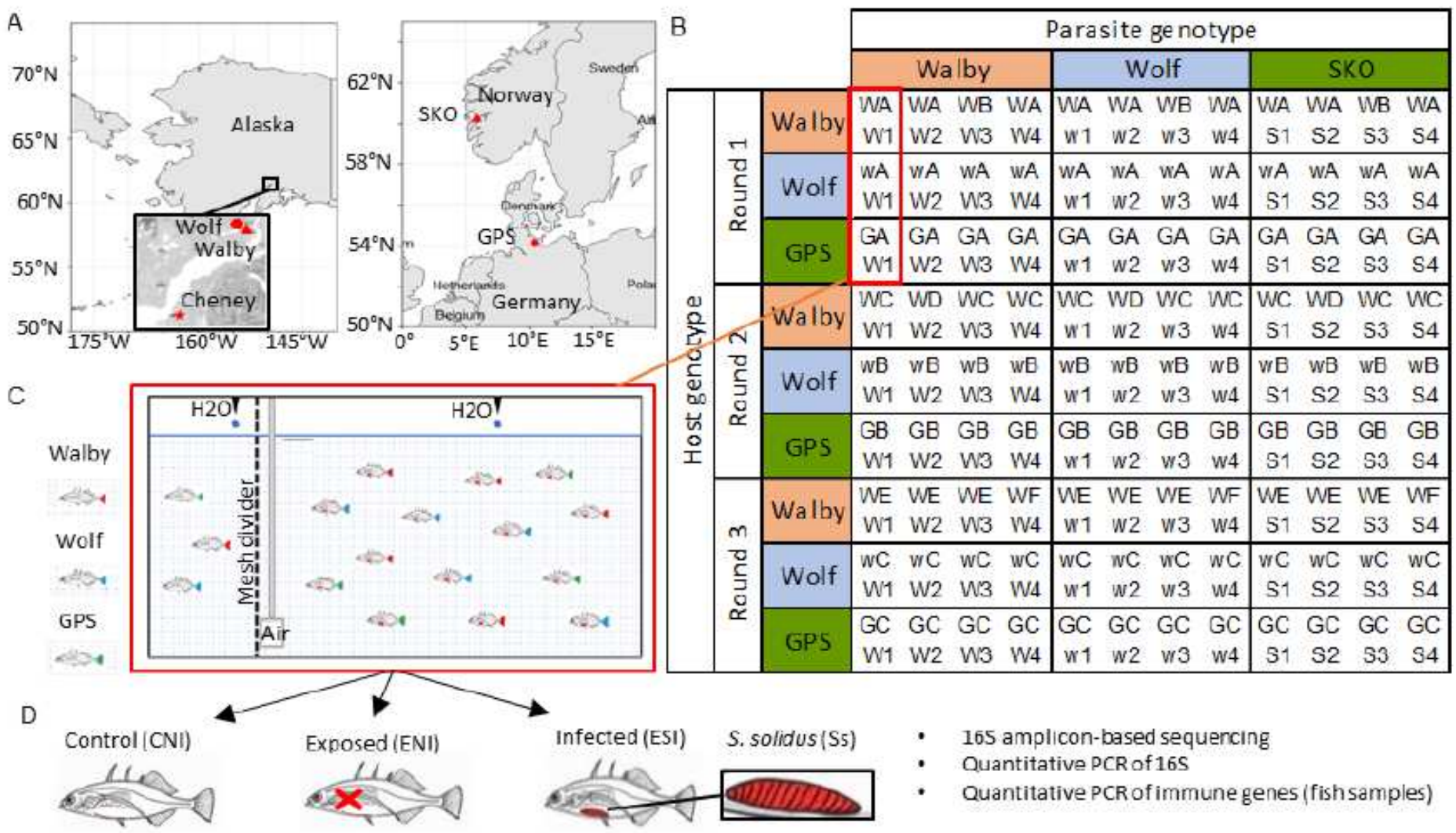

\section{Figure 1}

Summary of experimental design. (A) The map displays all sampling locations. The field study was conducted on sticklebacks and S. solidus from one location in Alaska (Cheney). The three genotypes of stickleback hosts (Walby (W), Wolf (w) and GPS(G)) and three genotypes of S. solidus (Walby (W), Wolf (w) and SKO (S)) used for experimental infection were collected from lakes in Alaska and Europe. (B) Design matrix showing the organization of rounds and tanks based on fish genotype (denoted by letters) and parasite genotype (denoted by numbers). A total of 36 tanks were used. One example is squared in red. (C) Each of the tanks contained three sham control fish (left) from Wolf (Red Tail), Walby (Green Tail), and GPS (Blue Tail) separated by a mesh divider from 5 Wolf fish , 5 GPS fish, and 4 Walby fish that had been exposed to parasites (right). (D) Upon dissection, the success of infection was assessed, and exposed individuals were classified as exposed non-infected (ENI) or exposed successfully infected (ESI). All successfully infected fish were processed, and corresponding ENI and control non-infected fish from the same tanks and fish origin were processed as controls. Note: The designations employed and the presentation of the material on this map do not imply the expression of any opinion whatsoever on the part of Research Square concerning the legal status of any country, territory, city or area or of its authorities, or concerning the delimitation of its frontiers or boundaries. This map has been provided by the authors. 

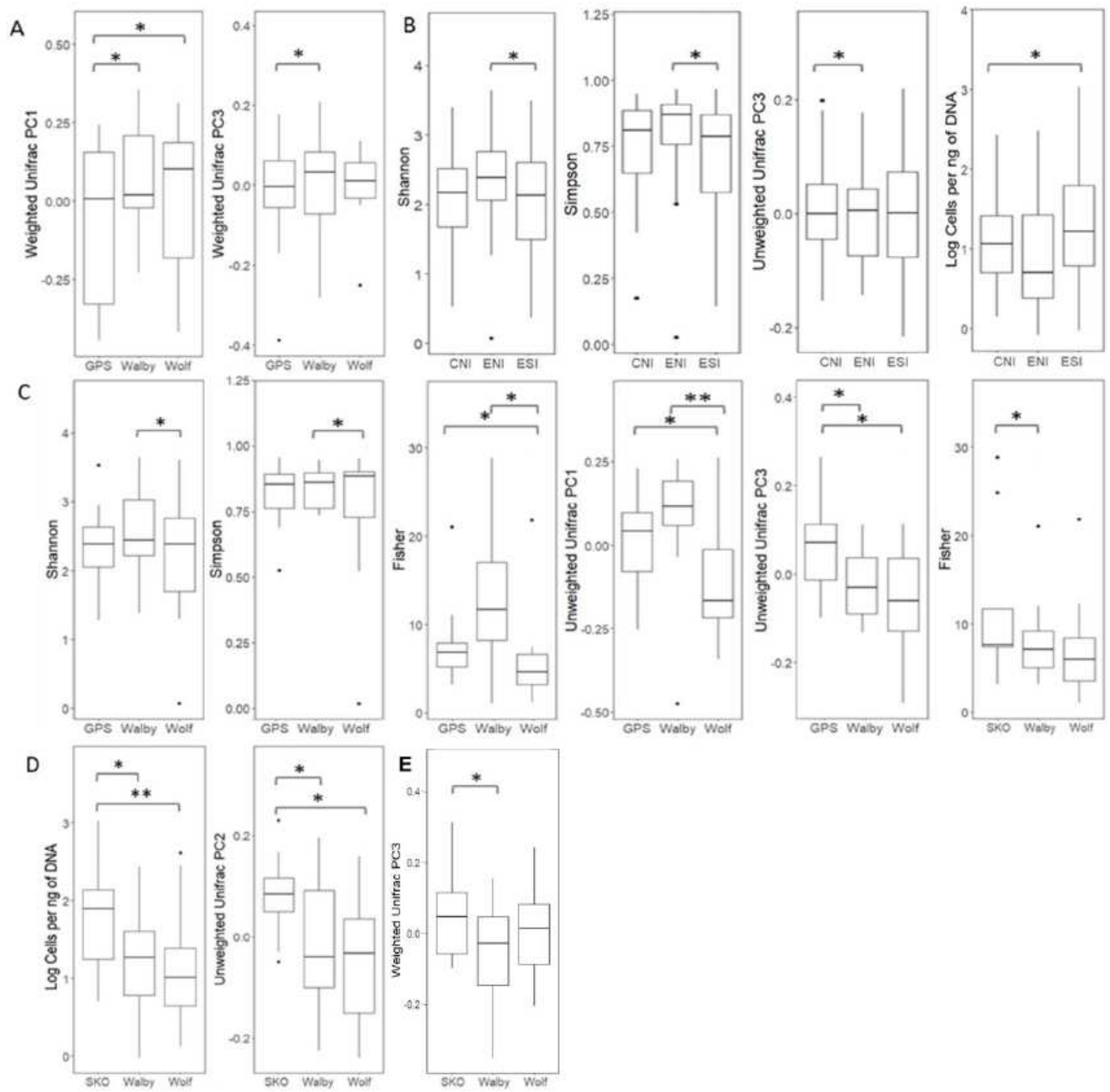

\section{Figure 2}

Stickleback bacterial community composition vary with exposure, infection status, host genotype and parasite genotype as revealed by LMMs. (A) Among the control non-infected fish, the microbiome composition varies with fish genotype. Differences were found between Walby $(n=17)$ and GPS fish $(n=15)$ (Weighted Unifrac, Axes 1 LMM $p=0.038$; and Axis 3, Unweighted Unifrac, Axis 3, $p=0.028$ ) and between Wolf $(n=10)$ and GPS fish (Unweighted Unifrac Axis 1, $p=0.024)$. (B) Exposure and infection impact the microbial diversity and composition. Exposure impacts beta diversity (Weighted Unifrac, Axis $3, p=0.031)$, whereas successful infection is associated with changes in bacterial load $(p=0.01)$ and 
alpha diversity (Shannon $p=0.039$, Simpson $p=0.013$ ). (C) Among exposed but non-infected fish, the microbiome diversity and composition vary with host genotype and parasite genotype. Differences were found between Wolf $(n=9)$ and GPS $(n=14)$ fish (Fisher $p=0.008$, Unweighted Unifrac Axis $1 p=0.005$, and $3 p=0.013)$, between Walby $(n=10)$ and Wolf fish (Shannon $p=0.034$, Simpson $p=0.020$, Fisher $p=0.004$, Unweighted Unifrac Axis $1 \mathrm{p}=0.006$ ) and between Walby and GPS fish (Unweighted Unifrac Axis 3 $p=0.041$ ). Differences were found between alpha diversity of fish exposed to Walby and SKO parasites (Fisher $p=0.018$ ). (D) Among infected sticklebacks, microbiome composition varies with parasite origin and host-parasite co-evolutionary history. Fish infected with SKO $(n=18)$ parasites had higher bacterial load than fish infected with Walby $(n=27, p=0.010)$ and Wolf parasites $(n=25, p=0.001)$. Unweighted Unifrac distance revealed distinct clustering of fish infected with SKO parasites and fish infected with Walby or Wolf parasites (Axis 2, $p=0.046$ and $p=0.047$ respectively). (E) Within parasites, microbiome composition varies with parasite origin. Weighted Unifrac distance revealed distinct clustering of the microbiome of SKO $(n=18)$ and Walby $(n=27)$ parasites (Axis 3, $p=0.035)$. The bottom and top edges of the boxes mark the 25th and 75th percentiles. The horizontal line denotes the median. Whiskers mark the range of the data excluding outliers (dots).

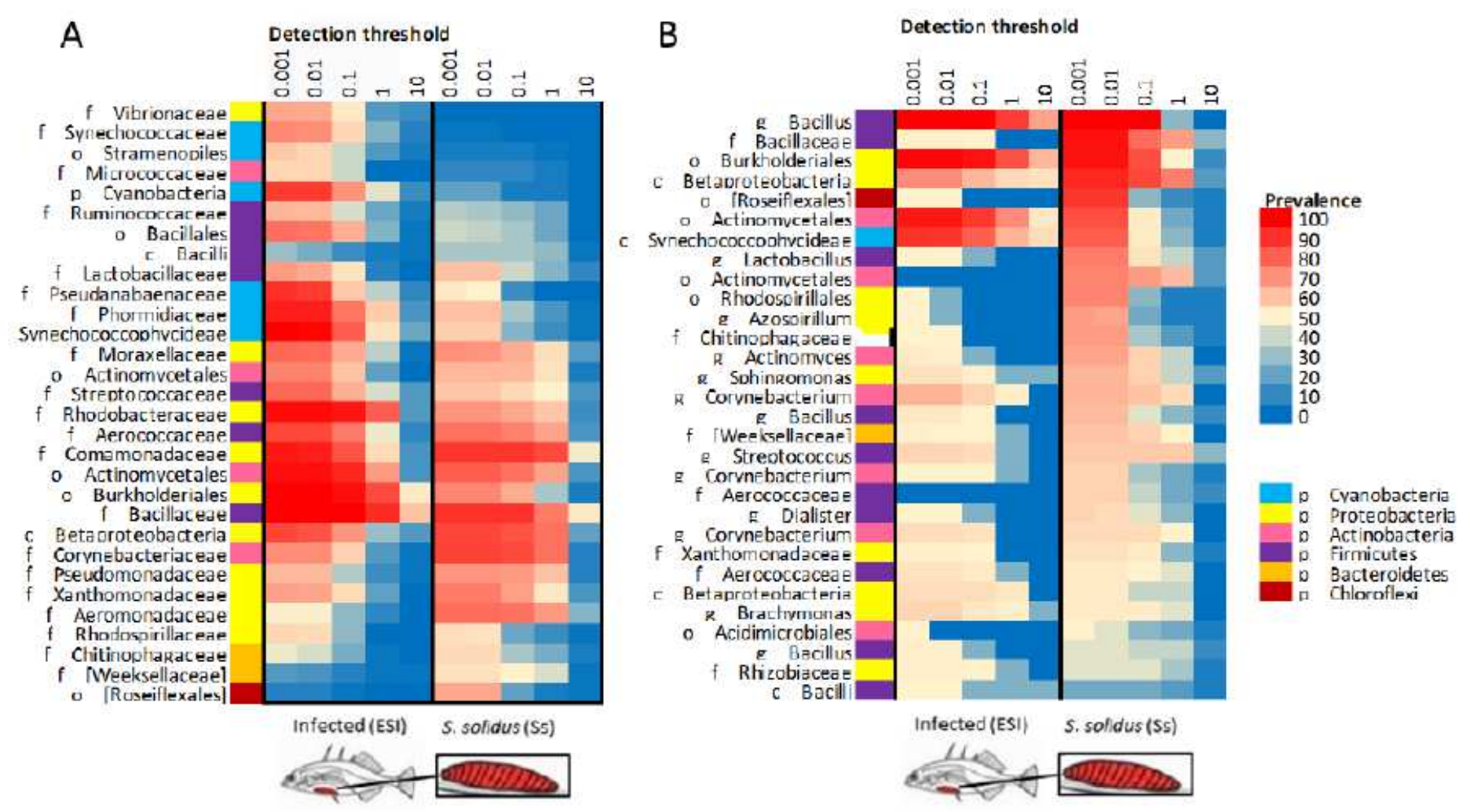

\section{Figure 3}

Bacterial taxa at multiple taxonomic levels vary in relative abundance and prevalence between threespine stickleback gut and S. solidus plerocercoids. The heatmaps depict the prevalence of the bacterial families (A) or ASVs (B) at different detection thresholds (defined in terms of minimum relative abundance) for successfully infected (ESI) sticklebacks, and corresponding S. solidus. A/ 30 most abundant bacterial 
families across all samples. B/ 30 most abundant ASVs in S. solidus. The phylum is provided as a color code next to the taxa name.
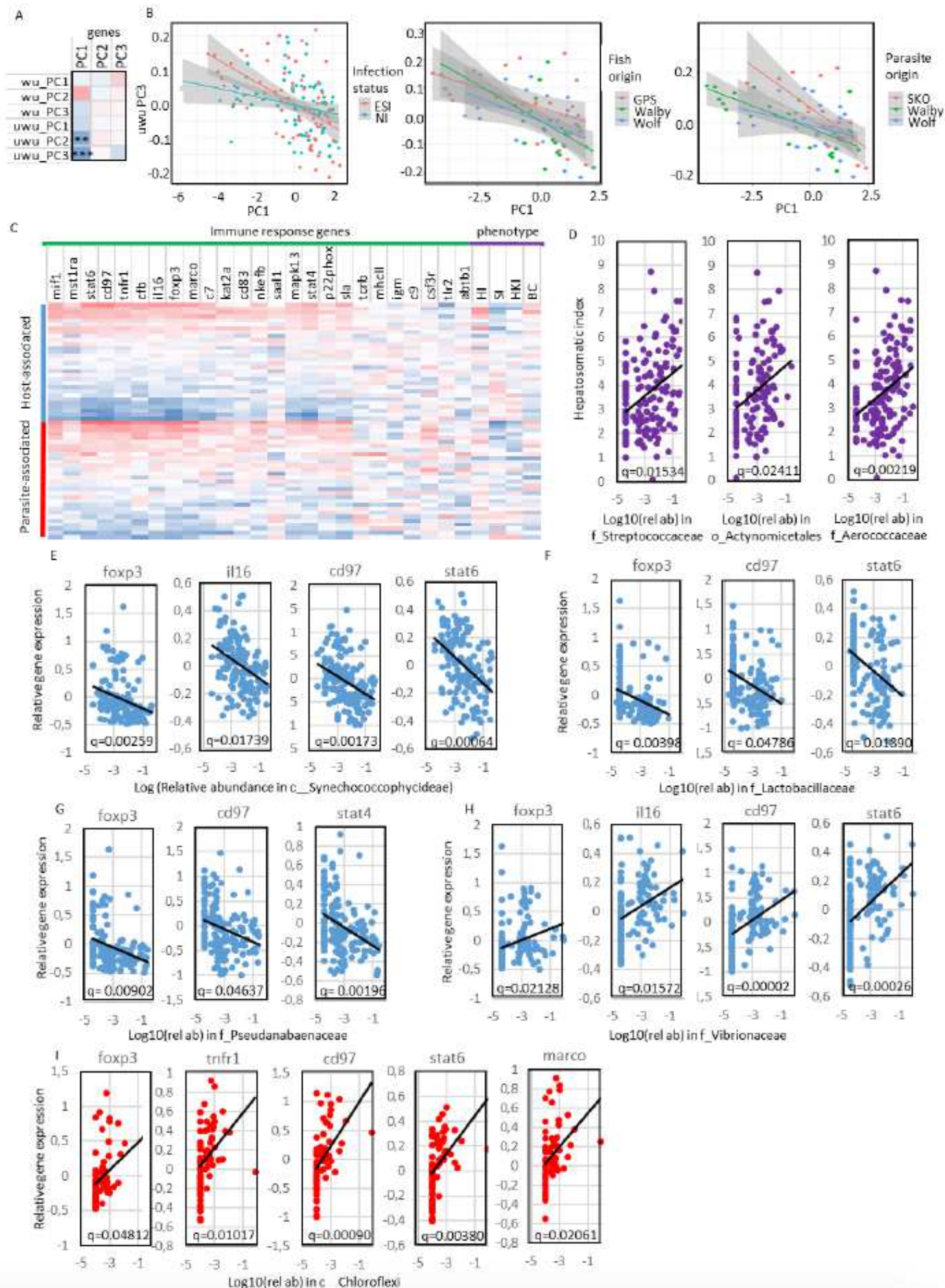

Figure 4

The host phenotype depends on the composition of the microbiome of the host and of its parasite. A. Correlation heatmap depicting gene expression - microbial profile correlations. Color of the squares indicate the strength of Spearman's rank correlation. Asterisks indicate significance of the correlation (* 
$\left.\mathrm{q}<0.05 ;{ }^{* *} \mathrm{q}<0.05 ;{ }^{* \star *} \mathrm{q}<0.001\right)$. B. Comparison of correlations between gene PC1 and uwuPC3 depending on infection status, host genotype and parasite genotype. Significant differences are observed when the confidence interval at the lower and/or higher values do not overlap with the mean of the other one. C. A. Correlation heatmap between the expression of genes, and phenotypic indices $(\mathrm{HI}$, Hepatosomatic index; SI, Splenosomatic index, HKI Head-kidney index; BC, Body condition) and the relative abundance in the most abundant acterial families in the host $\mathrm{G}$. aculeatus, and its parasite $\mathrm{S}$. solidus. Color indicate the strength of Spearman's rank correlation. The complete figure with indication of significance is available in Figure S12. Significant Spearman's rank correlation (with FDR $<0.05$ ) are plotted in (D) between the relative abundance in Streptococcaceae, an unknown Actynomicetales and Aerococcaceae in the host with Heptaosomatic index $(N=145)$, in $(E)$ between the relative abundance of Synechoccophycidae in fish and the relative expression of foxp3, il16, cd97 and stat6 (N=145), in ( $F)$ between the relative abundance in Lactobacillaceae in fish and the relative expression of foxp3, cd97 and stat6 $(N=145)$, in $(G)$ between the relative abundance in Pseudanabaenaceae in fish and the relative expression of foxp3, cd97 and stat4 $(\mathrm{N}=145)$, in $(\mathrm{H})$ between the relative abundance of Vibrionaceae in fish and relative expression of of foxp3, il16, cd97 and stat6 $(\mathrm{N}=145)$ and in (I) between the relative abundance of Chloroflexi in the parasite and relative abundance in foxp3, tnfr $1, \operatorname{cd} 97$, stat6, and marco $(\mathrm{N}=69)$. The significant Spearman correlation, after Bonferroni correction, are provided on each plot. 